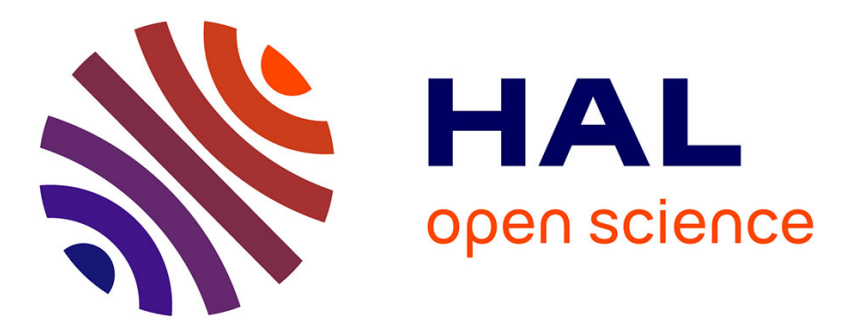

\title{
Role of nonconservative scattering forces and damping on Brownian particles in optical traps
}

Matthieu Mangeat, Yacine Amarouchene, Yann Louyer, Thomas Guérin, David S. Dean

\section{- To cite this version:}

Matthieu Mangeat, Yacine Amarouchene, Yann Louyer, Thomas Guérin, David S. Dean. Role of nonconservative scattering forces and damping on Brownian particles in optical traps. Physical Review E , 2019, 99 (5), 10.1103/PhysRevE.99.052107 . hal-02125304

\section{HAL Id: hal-02125304 \\ https://hal.science/hal-02125304}

Submitted on 10 May 2019

HAL is a multi-disciplinary open access archive for the deposit and dissemination of scientific research documents, whether they are published or not. The documents may come from teaching and research institutions in France or abroad, or from public or private research centers.
L'archive ouverte pluridisciplinaire HAL, est destinée au dépôt et à la diffusion de documents scientifiques de niveau recherche, publiés ou non, émanant des établissements d'enseignement et de recherche français ou étrangers, des laboratoires publics ou privés. 


\title{
Role of nonconservative scattering forces and damping on Brownian particles in optical traps
}

\author{
Matthieu Mangeat, Yacine Amarouchene, Yann Louyer, Thomas Guérin, and David S. Dean \\ Université de Bordeaux, CNRS, LOMA, UMR 5798, F-33400 Talence, France
}

(Received 24 September 2018; published 8 May 2019)

\begin{abstract}
We consider a model of a particle trapped in a harmonic optical trap but with the addition of a nonconservative radiation induced force. This model is known to correctly describe experimentally observed trapped particle statistics for a wide range of physical parameters, such as temperature and pressure. We theoretically analyze the effect of nonconservative force on the underlying steady state distribution as well as the power spectrum for the particle position. We compute perturbatively the probability distribution of the resulting nonequilibrium steady states for all dynamical regimes underdamped through to overdamped and give expressions for the associated currents in phase space (position and velocity). We also give the spectral density of the trapped particle's position in all dynamical regimes and for any value of the nonconservative force. Signatures of the presence of nonconservative forces are shown to be particularly strong for the underdamped regime at low frequencies.
\end{abstract}

DOI: 10.1103/PhysRevE.99.052107

\section{INTRODUCTION}

The seminal work of Ashkin in the 1970s instigated the development of optical traps [1]. Optical traps or tweezers are an invaluable tool for studying statistical mechanics, both in and out of equilibrium at the level of single particles. Moving the center of the optical trap allows one to measure very small forces in a wide range of systems, including colloids [2], biological systems (for instance, viruses, proteins, and biopolymers) [3], as well as dielectric and metallic nanoparticles [4]. The same kind of perturbation can be used to analyze nonequilibrium processes, for instance, optical tweezers have been used to demonstrate various nonequilibrium fluctuation theorems [5]. However, in such studies the optical trap does not simply furnish a simple potential, optical scattering forces actually generate a nonconservative component to the overall force exerted by the trap. Because of the presence of these scattering forces, even static optical traps represent nonequilibrium systems as the particles trapped within them are driven by the nonconservative forces and thus are not described by a Gibbs-Boltzmann probability distribution. For instance, it has been demonstrated [6-9] both theoretically and experimentally that scattering forces induce nonequilibrium currents, so called Brownian vortices, for trapped overdamped Brownian particles. At atmospheric pressures and near room temperature, the underlying dynamics of a trapped particle can be taken to be overdamped Brownian. However, optical traps operated at low pressure can probe dynamics where inertial effects become important and cross over to the underdamped regime. Clearly the current state of the art of the theory has to be modified to take into account the effects of inertia, and this is the goal of this paper. Using the model of a Gaussian optical trap in the presence of a simplified nonconservative force, we examine the impact of inertial effects across the whole regime between overdamped and underdamped Brownian motions.

First, a general theory for the probability distribution of the perturbed steady state due to a small nonconservative force is developed for general damped Brownian dynamics. The modification of the steady state probability distribution function for the full phase space in the optical model of Ref. [9] is found, and the resulting marginal probability distributions for the particle position and velocity are computed. We demonstrate that the Brownian vortices found in the current of the particle position persists and remains geometrically the same as that found in Ref. [9]. However, the amplitude of the vortices depends on the damping parameters, and we will show that this damping parameter can be tuned in order to optimize the nonequilibrium current. In addition, we show that there is a nonequilibrium current in the space of velocities; the vortex here turns out to be remarkably similar to the vortex in position space.

For the optical trap model of Ref. [9], we also compute the spectral density of the particle position for general damping. Our result recovers the computation of Ref. [10] for overdamped Brownian motion and generalizes it to all damping regimes. Here, we see that the nonconservative force generates very strong changes in the spectral density at low frequencies and that the amplitude of the low-frequency spectral density can be tuned by varying the friction coefficient $\gamma$ and that there is a critical value of $\gamma$ where it is minimized. Finally, we also derive a function correlation which is a signal of the breaking of time reversal symmetry due to the nonconservative optical scattering force.

Some of the results derived in this paper are used in an accompanying paper where the role of inertia in the presence of nonconservative forces is demonstrated in the experimental context [11]. In this paper, we concentrate on the presence of a nonconservative force in addition to a purely harmonic trap. This simplified model allows the derivation of a large number of analytical results. However, to fully explain the results of Ref. [11] where small anharmonicities can give rise to large effects in the underdamped regime due to near resonance phenomena, quartic terms in the potential must be considered to fully reproduce the experimental results. However, the results derived here can be adapted to the experimental context by using effective harmonic parameters describing the nonlinear 
system [12]. This therefore yields an analytic theory sufficient to completely describe the experiments and fully nonlinear numerical simulations [11].

\section{MODELING PARTICLES IN OPTICAL TRAPS}

\section{A. Langevin dynamics}

In this section, we first discuss the underlying Langevin dynamics used to model the trapped particle. We then discuss the underlying model for trapping, aimed at experts in nonequilibrium statistical mechanics who are perhaps not familiar with the physics of optical trapping.

In this paper, we will consider the dynamics of Brownian particles in an optical trap. If $\mathbf{x}(t)$ denotes the particle position, the underlying Langevin equation is

$$
m \frac{d^{2}}{d t^{2}} \mathbf{x}(t)=-\gamma \frac{d}{d t} \mathbf{x}(t)+\mathbf{F}(\mathbf{x})+\sqrt{2 T \gamma} \boldsymbol{\xi}(t),
$$

where $m$ is the particle mass, $\gamma$ is the friction coefficient, and $\mathbf{F}(\mathbf{x})$ is a time-independent force generated by the optical trap. The noise term is assumed to have Gaussian white noise correlations in time; this noise has zero mean and correlation function,

$$
\left\langle\xi_{i}(t) \xi_{j}\left(t^{\prime}\right)\right\rangle=\delta_{i j} \delta\left(t-t^{\prime}\right),
$$

in the amplitude, term $T$ is the temperature measured in units where $k_{B}=1$. When the trap is applied in a gas phase, the friction coefficient can be significantly modified, and one can go from the underdamped to overdamped regimes with the same experimental setup if one can control the pressure in the trapping cell.

A semiphenomenological relationship for the friction coefficient in air as a function of the pressure is given by $[13,14]$

$$
\gamma=6 \pi \eta_{\mathrm{atm}} R_{p} \frac{0.619}{0.619+\mathrm{Kn}}\left(1+c_{K}\right)
$$

where, in the first Stokes draglike term, $R_{p}$ is the radius of the particle and $\eta_{\mathrm{atm}}$ is the viscosity coefficient of air at atmospheric pressure. In addition, $\mathrm{Kn}$ is the Knudsen number, accounting from the deviation from continuum behavior $\mathrm{Kn}=$ $\lambda_{\mathrm{mfp}} / R_{p}$ where $\lambda_{\mathrm{mfp}}$ is the mean free path of air molecules and is given by $\lambda_{\mathrm{mfp}}=(68 \mathrm{~nm}) P_{\mathrm{atm}} / P$, where $P$ is the applied pressure in the trap and $P_{\text {atm }}$ is the atmospheric pressure. Finally $c_{K}=(0.31 \mathrm{Kn}) /\left(0.785+1.152 \mathrm{Kn}+\mathrm{Kn}^{2}\right)$. We thus see that the value of the friction coefficient can be tuned by modifying the pressure of the trap.

\section{B. Model of the optical trap}

In the experiment described in the accompanying paper [11], the wavelength of the laser is $\lambda=1064 \mathrm{~nm}$, whereas the radius of the trapped (fused silica) particle is $R_{p}=68 \mathrm{~nm}$. As $R_{p} \ll \lambda$, we are in the Rayleigh regime where the particle can be treated as a point dipole.

Here, for completeness, we review the model of the optical trap used in Ref. [15] as well as reviewing the underlying physics giving rise to both conservative and nonconservative forces in the dipole approximation [16]. The Lorentz force acting on an electric dipole $\mathbf{p}$ is given by

$$
\mathbf{F}=(\mathbf{p} \cdot \nabla) \mathbf{E}+\frac{d \mathbf{p}}{d t} \times \mathbf{B} .
$$

The response of the dipole to the applied electric field is taken to be linear,

$$
\mathbf{p}(t)=\int_{-\infty}^{\infty} d t^{\prime} \alpha\left(t-t^{\prime}\right) \mathbf{E}(\mathbf{r}, t)
$$

For a monochromatic electromagnetic field of angular frequency $\omega$ polarized in the $x$ direction, we can write

$$
\mathbf{E}(\mathbf{r}, t)=E_{0}(\mathbf{r}) \cos [\phi(\mathbf{r})-\omega t] \mathbf{e}_{x},
$$

where $\mathbf{e}_{x}$ denotes the unit vector in the direction $\mathbf{x}$ and $\phi(\mathbf{r})$ is the phase. Using the Maxwell equation $\partial \mathbf{B} / \partial t=-\nabla \times \mathbf{E}$, we can write

$$
\mathbf{B}=\frac{1}{\omega} \nabla \times\left\{E_{0}(\mathbf{r}) \sin [\phi(\mathbf{r})-\omega t] \mathbf{e}_{x}\right\} .
$$

Using linear response, we can write

$$
\begin{aligned}
\mathbf{p}(t)= & \operatorname{Re} \exp (-i \omega t) \int_{-\infty}^{\infty} d t^{\prime} \alpha\left(t-t^{\prime}\right) \mathbf{E}_{0}(\mathbf{r}) \exp [i \phi(\mathbf{r}) \\
& \left.-i \omega\left(t^{\prime}-t\right)\right] \\
= & \operatorname{Re} \mathbf{E}(\mathbf{r}, t) \alpha(\omega) \\
= & \left\{\alpha^{\prime}(\omega) \cos [\phi(\mathbf{r})-\omega t]-\alpha^{\prime \prime}(\omega) \sin [\phi(\mathbf{r})-\omega t]\right\} \mathbf{e}_{x},
\end{aligned}
$$

so $\alpha(\omega)$ is the polarizability at frequency $\omega$ and $\alpha^{\prime}$ and $\alpha^{\prime \prime}$ denote the real (dispersive) and imaginary (dissipative) parts, respectively. Now, evaluating the force and replacing the terms $\cos ^{2}[\phi(\mathbf{r})-\omega t]$ and $\sin ^{2}[\phi(\mathbf{r})-\omega t]$ by their temporal average $1 / 2$ and cross terms $\cos [\phi(\mathbf{r})-\omega t]$ and $\sin [\phi(\mathbf{r})-$ $\omega t$ ] by their temporal average 0 , we obtain

$$
\mathbf{F}=\frac{1}{4} \alpha^{\prime} \nabla I(\mathbf{r})+\frac{1}{2} \alpha^{\prime \prime} I(\mathbf{r}) \nabla \phi(\mathbf{r}),
$$

where $I(\mathbf{r})=|\mathbf{E}(\mathbf{r})|^{2}$ is the field intensity. The first term above is clearly a conservative force generated by the potential,

$$
V(\mathbf{r})=\frac{\alpha^{\prime}}{4} I(\mathbf{r})
$$

The second term contains, in general, a nonconservative component. Its underlying origin comes from the interaction of the magnetic field with the rate of change in the dipole moment.

The electric field produced by the laser is approximated by the Gaussian form

$$
\mathbf{E}(\mathbf{r})=E_{0}\left[1+\frac{z^{2}}{z_{0}^{2}}\right]^{-(1 / 2)} \exp \left[-\frac{x^{2}}{w_{x}^{2}(z)}-\frac{y^{2}}{w_{y}^{2}(z)}+i \phi(\mathbf{r})\right] \mathbf{e}_{\mathbf{x}},
$$

where the field is polarized in the direction $\mathbf{e}_{x}$ and for $\alpha=x, y$ we have

$$
w_{\alpha}(z)=w_{\alpha}\left[1+\frac{z^{2}}{z_{0}^{2}}\right]^{1 / 2}
$$


is the $z$-dependent beam radius. The phase is given by

$$
\phi(\mathbf{r})=k z-\arctan \left(\frac{z}{z_{0}}\right)+\frac{k\left(x^{2}+y^{2}\right)}{2 z\left(1+\frac{z_{0}^{2}}{z^{2}}\right)},
$$

where $k=2 \pi / \lambda$ is the wave vector. The parameter $z_{0}$ is the Rayleigh range and is normally used as a fitting parameter, however, it is predicted to be given by $z_{0} \sim \pi w_{\alpha}^{2} / \lambda$. Finally, the particle is assumed to be spherical, and the polarizability $\alpha$ is taken to be [16]

$$
\alpha=\frac{\alpha_{0}}{1-i \frac{k^{3} \alpha_{0}}{6 \pi \varepsilon_{0}}},
$$

where $\alpha_{0}$ is the zero-frequency polarizability given by

$$
\alpha_{0}=4 \pi \epsilon_{0} R_{p}^{3} \frac{\epsilon-1}{\epsilon+2},
$$

and $\epsilon_{0}$ is the dielectric constant of the vacuum whereas $\epsilon$ is the relative dielectric constant of the particle. The form of the electric field given by Eq. (11) arises from the paraxial approximation to Maxwell's equation for the electric field in the vacuum and, in this case, the corresponding Helmholtz equation. The paraxial approximation assumes that for the whole electric field function denoted by $E$ (polarized in the direction $x$ ) one has that $\left|\partial^{2} E / \partial z^{2}\right| \ll|k \partial E / \partial z|$. Inspection of Eqs. (11) and (13) shows that the paraxial approximation is valid when $z / z_{0}^{2} \ll k$ and one can expand the force perturba- tively for small $z$ when $z / z_{0} \ll 1$. The first inequality implies that we must have $z \ll 2 \pi^{3} \omega_{\alpha}^{4} / \lambda^{3}$.

In the accompanying paper [11], the laser waist parameters are estimated in the high pressure regime where both the effects of trap nonlinearities and the scattering forces are weak. It is found that $w_{x}=0.915, w_{y}=1.034$, and $z_{0}=$ $w_{z}=2.966 \mu \mathrm{m}$ (in the notation of Ref. [11]) and so we must have $z \ll 0.29 \mu \mathrm{m}$. In addition, one may also Taylor expand the forces when $x / w_{x}$ and $y / w_{z}$ are small, and experimental results show that these two values are both on the order of 0.1 , so whereas nonlinear effects are weak, they are not completely negligible.

Under the above assumptions, to lowest order, the potential part of the force is generated by a quadratic potential,

$$
V(\mathbf{r})=\kappa_{x} \frac{x^{2}}{2}+\kappa_{y} \frac{y^{2}}{2}+\kappa_{z} \frac{z^{2}}{2},
$$

with spring constants given by $\kappa_{x}=E_{0}^{2} \alpha^{\prime} / w_{x}^{2}, \kappa_{y}=$ $E_{0}^{2} \alpha^{\prime} / w_{y}^{2}$, and $\kappa_{z}=E_{0}^{2} \alpha^{\prime} / 2 z_{0}^{2}$.

The phase factor $\phi$ is given, expanding to first and next orders of the particle displacement by

$$
\phi(\mathbf{r})=z\left(k-\frac{1}{z_{0}}\right)+\frac{z}{z_{0}^{2}}\left[\frac{k}{2}\left(x^{2}+y^{2}\right)+\frac{z^{2}}{3 z_{0}}\right] .
$$

As mentioned above, the term $z_{0}$ is often treated as a fitting parameter as the solution Eq. (11) is an approximate solution. However, an alternative physical approach is to insist that the approximated solution obeys Maxwell's equations exactly near the center of the trap. Expanding the solution for small $x, y$, and $z$ up to cubic order, we find that

$$
E_{x}(\mathbf{r}) \approx E_{0}\left\{1-\frac{x^{2}}{w_{x}^{2}}-\frac{y^{2}}{w_{y}^{2}}-\frac{z^{2}}{2}\left(\frac{2}{z_{0}^{2}}+k^{2}-\frac{2 k}{z_{0}}\right)+i\left[z\left(k-\frac{1}{z_{0}}\right)+\frac{k z\left(x^{2}+y^{2}\right)}{2 z_{0}^{2}}+\frac{z^{3}}{3 z_{0}^{3}}-\frac{z^{3}}{6}\left(k-\frac{1}{z_{0}}\right)^{3}\right]\right\} .
$$

Now, using the Helmholtz equation $\nabla^{2} E_{x}+k^{2} E_{x}=0$ near the origin, we find the $O(0)$ equation, coming from the real term,

$$
\frac{1}{z_{0}^{2}}-\frac{k}{z_{0}}+\frac{1}{w_{x}^{2}}+\frac{1}{w_{y}^{2}}=0
$$

giving an exact relation between $z_{0}$ and the two waists $w_{x}$ and $w_{y}$ in terms of the wave vector $k$. In the case where $w_{x}=w_{y}=w$ and when $k \gg 1 / z_{0}$ (so we assume that the paraxial approximation is valid), we obtain the standard formula $z_{0}=w^{2} k / 2=$ $\pi w^{2} / \lambda$. However, this formula is only valid if $2 \pi^{2} w^{2} / \lambda^{2} \gg 1$. Taking the root of Eq. (19) compatible with the standard formula, we find

$$
z_{0}=\frac{2}{k\left[1-\left(1-\frac{4}{k^{2} w_{x}^{2}}-\frac{4}{k^{2} w_{y}^{2}}\right)^{1 / 2}\right]},
$$

or in terms of the wavelength $\lambda$, we have

$$
z_{0}=\frac{\lambda}{\pi}\left[1-\left(1-\frac{\lambda^{2}}{\pi^{2} w_{x}^{2}}-\frac{\lambda^{2}}{\pi^{2} w_{y}^{2}}\right)^{1 / 2}\right]^{-1} .
$$

Using this to determine $z_{0}$ with the values of $w_{x}$ and $w_{y}$ determined above yields $z_{0}=2.781 \mu \mathrm{m}$, which is different from the fully fitted value of $z_{0}$ by about $6 \%$. This estimate thus provides a useful starting point to determine the trap parameters in the fitting procedure.

The scattering part of the force is given to leading quadratic order by

$$
\mathbf{F}_{\text {scat }}=\frac{\alpha^{\prime \prime}}{2} E_{0}^{2}\left\{\mathbf{e}_{x} \frac{k x z}{z_{0}^{2}}+\mathbf{e}_{y} \frac{k y z}{z_{0}^{2}}+\mathbf{e}_{z}\left[\left(k-\frac{1}{z_{0}}\right)\left(1-\frac{2 x^{2}}{w_{x}^{2}}-\frac{2 y^{2}}{w_{y}^{2}}-\frac{z^{2}}{z_{0}^{2}}\right)+k \frac{x^{2}+y^{2}}{2 z_{0}^{2}}+\frac{z^{2}}{z_{0}^{3}}\right]\right\},
$$


which can be written in terms of the spring constant $\kappa_{z}$ as

$$
\mathbf{F}_{\text {scat }}=\frac{\alpha^{\prime \prime}}{\alpha^{\prime}} \kappa_{z}\left\{\mathbf{e}_{x} k x z+\mathbf{e}_{y} k y z+\mathbf{e}_{z}\left[z_{0}\left(z_{0} k-1\right)\left(1-\frac{2 x^{2}}{w_{x}^{2}}-\frac{2 y^{2}}{w_{y}^{2}}-\frac{z^{2}}{z_{0}^{2}}\right)+k \frac{x^{2}+y^{2}}{2}+\frac{z^{2}}{z_{0}}\right]\right\},
$$

which gives

$$
\mathbf{F}_{\text {scat }}=\frac{\alpha^{\prime \prime}}{\alpha^{\prime}} \kappa_{z}\left\{\mathbf{e}_{x} k x z+\mathbf{e}_{y} k y z+\mathbf{e}_{z}\left[z_{0}\left(z_{0} k-1\right)-x^{2}\left(\frac{2 z_{0}\left(z_{0} k-1\right)}{w_{x}^{2}}-\frac{k}{2}\right)-y^{2}\left(\frac{2 z_{0}\left(z_{0} k-1\right)}{w_{y}^{2}}-\frac{k}{2}\right)-z^{2}\left(k-\frac{2}{z_{0}}\right)\right]\right\} .
$$

The lowest order approximation that takes into account both conservative-potential and scattering forces is thus the expressions for $\mathbf{F}_{p}=-\nabla V[O(1)]$ and $\mathbf{F}_{\text {scat }}[O(0)+O(2)]$. The next order corrections are $O(3)$ terms to $\mathbf{F}_{p}$ coming from anharmonic contributions.

Using the form Eq. (11) in the Helmholtz equation without making any approximations yields

$$
\nabla^{2} E_{x}-[\nabla \phi]^{2} E_{x}+k^{2} E_{x}+i\left(2 \nabla E_{x} \cdot \nabla \phi+E_{x} \nabla^{2} \phi\right)=0 .
$$

The real and imaginary parts of the above give two equations. The scattering force is given by

$$
\mathbf{F}_{\mathrm{scat}}=\frac{\alpha^{\prime \prime}}{2} I \nabla \phi=\frac{\alpha^{\prime \prime}}{2} E_{x}^{2} \nabla \phi,
$$

and taking the divergence of this yields

$$
\nabla \cdot \mathbf{F}_{\text {scat }}=\frac{\alpha^{\prime \prime}}{2} E_{x}\left(2 \nabla E_{x} \cdot \nabla \phi+E \nabla^{2} \phi\right)=0,
$$

from Eq. (25). However, the result Eq. (24) gives $\boldsymbol{\nabla} \cdot \mathbf{F}_{\text {scat }}=$ $4 z \alpha^{\prime \prime} \kappa_{z} / \alpha^{\prime} z_{0}$. In the limit $z_{0} \rightarrow \infty$, where the paraxial approximation becomes exact we find that this term is zero, but, in general, it is not. This actually means that the very last term in Eq. (24) should not be there. This can be regarded as being due to an error in the phase term.

Taking this into account we can naturally divide the scattering force into two components,

$$
\mathbf{F}_{\text {scat }}=\mathbf{F}_{\text {scat }, 1}+\mathbf{F}_{\text {scat }, 2}
$$

where

$$
\mathbf{F}_{\text {scat }, 1}=\frac{\alpha^{\prime \prime}}{\alpha^{\prime}} \kappa_{z} z_{0}\left(z_{0} k-1\right)\left[1-\frac{2 x^{2}}{w_{x}^{2}}-\frac{2 y^{2}}{w_{y}^{2}}\right] \mathbf{e}_{z}
$$

and

$$
\mathbf{F}_{\text {scat }, 2}=\frac{\alpha^{\prime \prime}}{\alpha^{\prime}} k \kappa_{z}\left[\mathbf{e}_{x} x z+\mathbf{e}_{y} y z-\mathbf{e}_{z} z^{2}\right]
$$

For the values of $z_{0}$ obtained here the dominant scattering force is $\mathbf{F}_{\text {scat }, 1}$, however, as $z$ scales as $z_{0}$ when just the harmonic confinement is taken into account it is not evident that the last term in the direction $\mathbf{e}_{z}$ can be neglected. However, we find that numerically this turns out to be the case.

\section{PERTURBATIVE CALCULATION OF THE NONEQUILIBRIUM STEADY STATE PROBABILITY DENSITY FUNCTION AND CURRENT}

In the standard paradigm of statistical physics, Brownian particles are subjected to forces generated by potentials and this means that the underlying steady state distribution is given by the Gibbs-Boltzmann distribution. The crucial point here is that the steady state in the presence of a nonconservative force has an associated current. We note that Brownian particles subject to linear potentials in unbounded systems also have currents, however, because the system is unbounded the probability distribution spreads with time and can be associated with effective transport coefficients [17]. However, in periodic systems, the particle position modulo of the period of the system does have a steady state which carries a current, and this system can be analyzed for overdamped Brownian particles [18]. In general, very few results exist on the steady states of systems with nonconservative forces or in the presence of driving (even for trapped monoparticle systems where only one body forces are present). Here, we will resort to the use of perturbation theory, which can be justified when the nonconservative component of the force is weak in comparison to the conservative component. Indeed, perturbation theory was the approach employed in Ref. [9] to analyze a trapped overdamped Brownian particle.

We will consider systems which are driven by underlying white noise and thus include both underdamped and overdamped Brownian motions. The probability distribution of the particle's position in phase space is thus described by the Fokker-Planck equation,

$$
\frac{\partial P(\mathbf{y})}{\partial t}=-H P
$$

where $\mathbf{y}$ denotes the phase-space coordinates and $H$ is the forward Fokker-Planck operator. Before perturbation by a nonconservative force, we assume that the system obeys detailed balance and has an underlying Gibbs-Boltzmann distribution denoted by $P_{0}$, obeying

$$
H P_{0}=0 .
$$

Now, we consider what happens when the system is perturbed by an additional force which is in terms of the Fokker-Planck operator denoted by the perturbation $\Delta H$. The question we address is what is the new steady state distribution $P_{s}$ defined by

$$
[H+\Delta H] P_{s}=0 .
$$

To first order in perturbation theory, we write $P_{s}=P_{0}+\Delta P_{0}$ where $\Delta P_{0}$ is given by

$$
H \Delta P_{0}=-\triangle H P_{0}
$$

and we refer to $\Delta P_{0}$ as the shift in the steady state probability distribution due to the additional force. Equation (34) has the formal solution,

$$
\Delta P_{0}(\mathbf{y})=-\int d \mathbf{y}^{\prime} H^{-1}\left(\mathbf{y}, \mathbf{y}^{\prime}\right)\left[\Delta H P_{0}\right]\left(\mathbf{y}^{\prime}\right) .
$$


Here, $H^{-1}$ is the pseudo-Green's function for the operator $H$ as the solution $\Delta H P_{0}$ must obey the normalization,

$$
\int d \mathbf{y} \Delta P_{0}(\mathbf{y})=0 .
$$

The transition probability $P\left(\mathbf{y} \mid \mathbf{y}^{\prime} ; t\right)$ can formally be written as

$$
P\left(\mathbf{y} \mid \mathbf{y}^{\prime} ; t\right)=\exp (-t H)\left(\mathbf{y}, \mathbf{y}^{\prime}\right)
$$

and the pseudo-Green's function thus as

$$
H^{-1}\left(\mathbf{y}, \mathbf{y}^{\prime}\right)=\int_{0}^{\infty} d t\left[P\left(\mathbf{y} \mid \mathbf{y}^{\prime} ; t\right)-P_{0}(\mathbf{y})\right] .
$$

This yields

$$
\Delta P_{0}(\mathbf{y})=-\int_{0}^{\infty} d t \int d \mathbf{y}^{\prime}\left[P\left(\mathbf{y} \mid \mathbf{y}^{\prime} ; t\right)-P_{0}(\mathbf{y})\right]\left[\Delta H P_{0}\right]\left(\mathbf{y}^{\prime}\right)
$$

Now, using the time reversal symmetry of the equilibrium state,

$$
P\left(\mathbf{y} \mid \mathbf{y}^{\prime} ; t\right)=\frac{P\left(\mathbf{y}^{\prime *} \mid \mathbf{y}^{*} ; t\right) P_{0}\left(\mathbf{y}^{*}\right)}{P_{0}\left(\mathbf{y}^{\prime}\right)},
$$

where ${ }^{*}$ indicates time reversal of the coordinates, this means that coordinates with an odd number of temporal derivatives change sign. Taking $t=0$, we see that time reversal symmetry also implies that $P_{0}\left(\mathbf{y}^{*}\right)=P_{0}(\mathbf{y})$. For overdamped Brownian particles, this has no effect as the position vector obeys $\mathbf{X}=$ $\mathbf{X}^{*}$. However, if $\mathbf{Y}$ represents the position and velocity $\mathbf{Y}=$ $(\mathbf{X}, \mathbf{V})$, we have $\mathbf{Y}^{*}=(\mathbf{X},-\mathbf{V})$. We thus find

$$
\begin{aligned}
\Delta P_{0}(\mathbf{y})= & -P_{0}(\mathbf{y}) \int_{0}^{\infty} d t \int d \mathbf{y}^{\prime}\left[P\left(\mathbf{y}^{\prime *} \mid \mathbf{y}^{*} ; t\right)-P_{0}\left(\mathbf{y}^{\prime}\right)\right] \\
& \times \frac{\left[\Delta H P_{0}\right]\left(\mathbf{y}^{\prime}\right)}{P_{0}\left(\mathbf{y}^{\prime}\right)}
\end{aligned}
$$

If the stochastic process represented by the Fokker-Planck equation is denoted by $\mathbf{Y}_{t}$ and we use the notation,

$$
\left\langle f\left(\mathbf{Y}_{t}\right)\right\rangle_{\mathbf{y}}=\int d \mathbf{y}^{\prime} P\left(\mathbf{y}^{\prime} \mid \mathbf{y} ; t\right) f\left(\mathbf{y}^{\prime}\right),
$$

i.e., the average value of $f\left(\mathbf{Y}_{t}\right)$ at time $t$ given that $\mathbf{Y}_{0}=\mathbf{y}$, and

$$
\left\langle f\left(\mathbf{Y}_{t}\right)\right\rangle_{0}=\int d \mathbf{y}^{\prime} P_{0}(\mathbf{y}) f\left(\mathbf{y}^{\prime}\right),
$$

the equilibrium value of $f(\mathbf{Y})$. We thus change the integration variable to write

$$
\begin{aligned}
\Delta P_{0}(\mathbf{y})= & -P_{0}(\mathbf{y}) \int_{0}^{\infty} d t \int d \mathbf{y}^{\prime}\left[P\left(\mathbf{y}^{\prime} \mid \mathbf{y}^{*} ; t\right)-P_{0}\left(\mathbf{y}^{\prime}\right)\right] \\
& \times \frac{\left[\Delta H P_{0}\right]\left(\mathbf{y}^{\prime *}\right)}{P_{0}\left(\mathbf{y}^{\prime *}\right)} .
\end{aligned}
$$

where we have used $\mathbf{y}^{* *}=\mathbf{y}$. This then yields

$$
\begin{aligned}
\Delta P_{0}(\mathbf{y})= & -P_{0}(\mathbf{y}) \int_{0}^{\infty} d t\left[\left\langle\frac{\left[\Delta H P_{0}\right]\left(\mathbf{Y}_{t}^{*}\right)}{P_{0}\left(\mathbf{Y}_{t}^{*}\right)}\right\rangle_{\mathbf{y}^{*}}\right. \\
& \left.-\left\langle\frac{\left[\Delta H P_{0}\right]\left(\mathbf{Y}^{*}\right)}{P_{0}\left(\mathbf{Y}^{*}\right)}\right\rangle_{0}\right] .
\end{aligned}
$$

The change in the equilibrium distribution at point $\mathbf{y}$ in phase space is thus given by a Kubo-type formula [19]. This result is general and applies to any system described by a FokkerPlanck equation.

\section{A. Overdamped Brownian motion}

Here, we consider a process characterized solely by the position $\mathbf{X}$ that is to say the overdamped regimes. Here, we assume that the unperturbed Fokker-Planck operator, defined via its action on a test function $f$, is given by

$$
H f=-\nabla \cdot D[\nabla f+\beta f \nabla V],
$$

i.e., it describes a particle with diffusivity $D$ in a potential $V$ at temperature $T$ (where, for convenience, we use units where $k_{B}=1$, the results for $T$ measured in kelvins are thus obtained by replacing $T$ by $k_{B} T$ in all the formulas which follow) and $\beta=1 / T$.

Here, the steady state is given by the Gibbs-Boltzmann equilibrium distribution,

$$
P_{0}(\mathbf{x})=\frac{1}{Z} \exp [-\beta V(\mathbf{x})]
$$

The perturbation of the Fokker-Planck operator due to the presence of an extra force $\mathbf{F}$ is given by

$$
\Delta H f=\nabla \cdot D \beta \mathbf{F} f .
$$

From this, we find

$$
\frac{\left[\Delta H P_{0}\right](\mathbf{x})}{P_{0}(\mathbf{x})}=-\beta^{2} D \nabla V(\mathbf{x}) \cdot \mathbf{F}(\mathbf{x})+\beta D \nabla \cdot \mathbf{F} .
$$

This now gives

$$
\begin{aligned}
\Delta P_{0}(\mathbf{x})= & P_{0}(\mathbf{x}) \beta \int_{0}^{\infty} d t\left[\left\langle\beta D \nabla V\left(\mathbf{X}_{t}\right) \cdot \mathbf{F}\left(\mathbf{X}_{t}\right)-D \nabla \cdot \mathbf{F}\left(\mathbf{X}_{t}\right)\right\rangle_{\mathbf{x}}\right. \\
& \left.-\langle\beta D \nabla V(\mathbf{X}) \cdot \mathbf{F}(\mathbf{X})+D \nabla \cdot \mathbf{F}(\mathbf{X})\rangle_{0}\right] .
\end{aligned}
$$

The case where the force is derived from a potential $\mathbf{F}=$ $-\nabla U$ should, of course, be simple as we know the new equilibrium measure should be

$$
P_{s}(\mathbf{x})=\frac{\exp [-\beta V(\mathbf{x})-\beta U(\mathbf{x})]}{\int d \mathbf{x} \exp [-\beta V(\mathbf{x})-\beta U(\mathbf{x})]},
$$

and for small $U$ should find

$$
P_{s}(\mathbf{x})=P_{0}(\mathbf{x})\left[1-\beta U(\mathbf{x})+\beta\langle U(\mathbf{X})\rangle_{0}\right] .
$$

It is illuminating as well as a useful check to obtain this from the general formalism derived previously. To do this, we use the following result of stochastic calculus [20] for an arbitrary function $U$ :

$$
\left\langle\frac{d U\left(\mathbf{X}_{\mathbf{t}}\right)}{d t}\right\rangle=-D \beta \nabla U\left(\mathbf{X}_{\mathbf{t}}\right) \cdot \nabla V\left(\mathbf{X}_{\mathbf{t}}\right)+D \nabla^{2} U\left(\mathbf{X}_{t}\right) .
$$

Using this and the relation $\mathbf{F}=-\nabla U$ in Eq. (50) now gives

$$
\Delta P_{0}(\mathbf{x})=P_{0}(\mathbf{y}) \beta \int_{0}^{\infty} d t\left[\left\langle\frac{d U\left(\mathbf{X}_{\mathbf{t}}\right)}{d t}\right\rangle_{\mathbf{x}}-\left\langle\frac{d U(\mathbf{X})}{d t}\right\rangle_{0}\right]
$$

Clearly, we have

$$
\left\langle\frac{d U(\mathbf{X})}{d t}\right\rangle_{0}=0
$$


and we have by definition that

$$
\lim _{t \rightarrow \infty}\left\langle U\left(\mathbf{X}_{t}\right)\right\rangle_{\mathbf{x}}=\langle U(\mathbf{X})\rangle_{0} .
$$

The time integral in Eq. (54) can now be simply carried out to yield

$$
\Delta P_{0}(\mathbf{x})=P_{0}(\mathbf{x})\left[-\beta U(\mathbf{x})+\beta\langle U(\mathbf{X})\rangle_{0}\right],
$$

in agreement with Eq. (52).

In general, the perturbing force can be decomposed into a conservative and nonconservative part via the Helmholtz decomposition where one writes

$$
\mathbf{F}=-\nabla U+\nabla \times \mathbf{A},
$$

where $U$ is determined from the equation,

$$
\nabla^{2} U=-\nabla \cdot \mathbf{F},
$$

and the remaining term can be similarly evaluated to write

$$
\mathbf{F}=-\nabla U+\mathbf{F}_{n}
$$

where the nonconservative part of the force $\mathbf{F}_{n}$ is given by

$$
\mathbf{F}_{n}=\mathbf{F}+\nabla U,
$$

however, the solution for $U$ must be chosen so that $\nabla \cdot \mathbf{F}_{n}=0$. Using this decomposition then yields

$$
\begin{aligned}
\Delta P_{0}(\mathbf{x})= & \beta P_{0}(\mathbf{x})\left[-U(\mathbf{x})+\langle U(\mathbf{X})\rangle_{0}\right. \\
& +\int_{0}^{\infty} d t\left[\left\langle\beta D \nabla V\left(\mathbf{X}_{t}\right) \cdot \mathbf{F}_{n}\left(\mathbf{X}_{t}\right)\right\rangle_{\mathbf{x}}\right. \\
& \left.\left.-\left\langle\beta D \nabla V(\mathbf{X}) \cdot \mathbf{F}_{n}(\mathbf{X})\right\rangle_{0}\right]\right] .
\end{aligned}
$$

The modification of the steady state probability density function by the nonconservative force is thus seen to be related to the work performed by the nonconservative force in the direction of the original potential $V$. This formula is not particularly illuminating from a physical point of view for overdamped Brownian motion, and when we study the case of general damping, we will find a much more explicit interpretation.

\section{Application to a model for an optical trap}

Here, we study the model proposed by Moyses et al. [9], where

$$
V(\mathbf{x})=\frac{\kappa}{2}\left(x^{2}+y^{2}+\eta z^{2}\right)
$$

so this represents an anisotropic harmonic trap unless $\eta=$ 1. We have seen that the potential part of the scattering force in Eq. (16), in general, also has $\kappa_{x} \neq \kappa_{y}$; however, as the difference between $\kappa_{x}$ and $\kappa_{y}$ is numerically small, we consider this case to simplify the notation and the subsequent analysis. In Ref. [9], the nonconservative component of the force generated by the optical trap is taken to be

$$
\mathbf{F}=\varepsilon \kappa a\left(1-\frac{x^{2}+y^{2}}{a^{2}}\right) \mathbf{e}_{z},
$$

$a$ is proportional to the waist $w_{x} \approx w_{y}$ (we use the same notation as Ref. [9] for ease of comparison). The part of the scattering force used is thus $\mathbf{F}_{\text {scat, } 1}$, expressed in Eq. (29), which is that which is numerically the most important. However, the nature of our perturbative computation means that the contributions to $\Delta P_{0}$ from each component of the nonconservative force is additive, and so we can simply compute the perturbations due to each component and then sum them. The perturbing parameter $\varepsilon$ is dimensionless, and we will compute the modified steady state probability density function to first order in $\varepsilon$.

The decomposition of the perturbation in terms of conservative and nonconservative contributions now yields the potential,

$$
U(\mathbf{x})=-\varepsilon \kappa a z,
$$

and the nonconservative component of the force,

$$
\mathbf{F}_{n}=-\varepsilon \kappa \frac{x^{2}+y^{2}}{a} \mathbf{e}_{z}
$$

Using this, we find

$$
\nabla V \cdot \mathbf{F}_{n}=-\varepsilon \kappa^{2} \eta z \frac{x^{2}+y^{2}}{a} .
$$

The evaluations of the expectation values required to compute the renormalization of the steady state probability distribution can be carried out by explicitly solving the Langevin equation for the unperturbed process; the corresponding equations are

$$
\begin{aligned}
& \dot{x}(t)=-\beta D \kappa x(t)+\sqrt{2 D} \xi_{x}(t), \\
& \dot{y}(t)=-\beta D \kappa y(t)+\sqrt{2 D} \xi_{y}(t), \\
& \dot{z}(t)=-\beta D \kappa \eta z(t)+\sqrt{2 D} \xi_{z}(t),
\end{aligned}
$$

where $\xi_{x}(t), \xi_{y}(t)$, and $\xi_{z}(t)$ are independent Gaussian white noises. The above equations can be solved in terms of the particle positions at $t=0$, denoted by $x, y$, and $z$ to give

$$
\begin{aligned}
& x(t)=\exp (-\beta D \kappa t) x+\sqrt{2 D} \exp (-\beta D \kappa t) \int_{0}^{t} d s \exp (\beta D \kappa s) \xi_{x}(s), \\
& y(t)=\exp (-\beta D \kappa t) y+\sqrt{2 D} \exp (-\beta D \kappa t) \int_{0}^{t} d s \exp (\beta D \kappa s) \xi_{y}(s), \\
& z(t)=\exp (-\beta D \kappa \eta t) z+\sqrt{2 D} \exp (-\beta D \kappa \eta t) \int_{0}^{t} d s \exp (\beta D \kappa \eta s) \xi_{z}(s) .
\end{aligned}
$$


Using these solutions, we find

$$
\left\langle\nabla V \cdot \mathbf{F}_{n}\right\rangle_{\mathbf{x}}=-\varepsilon \kappa^{2} \eta \exp (-\beta D \kappa \eta t) \times z \frac{\left(x^{2}+y^{2}\right) \exp (-2 \beta D \kappa t)+\frac{2}{\kappa \beta}[1-\exp (-2 \beta D \kappa t)]}{a},
$$

and we also see that

$$
\left\langle\nabla V \cdot \mathbf{F}_{n}\right\rangle_{0}=0 .
$$

Putting all this together [and noting that $\langle U(\mathbf{X})\rangle_{0}=0$ ], we obtain

$$
\begin{aligned}
\frac{\Delta P_{0}(\mathbf{x})}{P_{0}(\mathbf{x})}= & -\beta \Delta \phi=\frac{\varepsilon z}{a}\left[\beta \kappa a^{2}-2+\frac{\eta}{\eta+2}\right. \\
& \left.\times\left(2-\beta \kappa\left[x^{2}+y^{2}\right]\right)\right],
\end{aligned}
$$

where $\Delta \phi$ is interpreted as the renormalization of the trapping potential. This agrees with the result found in Ref. [9] for the same trapping potential and nonconservative perturbation considered here.

\section{Alternative formulation for overdamped particles}

The probabilistically based solution for the perturbed steady state distribution gives us an interpretation of the perturbation of the steady state in terms of work performed by the perturbing forces. However, in the case where the unperturbed solution is Gaussian, we see that the solution is particularly simple, the solution having a polynomial form for a polynomial perturbing force. Given the structure of the solution found, we make the same ansatz as in Ref. [9],

$$
\Delta P_{0}(\mathbf{x})=P_{0}(\mathbf{x}) K(\mathbf{x}),
$$

this yields

$$
\nabla^{2} K(\mathbf{x})-\beta \nabla V(\mathbf{x}) \cdot \nabla K(\mathbf{x})=\beta \nabla \cdot \mathbf{F}(\mathbf{x})-\beta^{2} \nabla V(\mathbf{x}) \cdot \mathbf{F}(\mathbf{x}) .
$$

For the case studied above as defined by the potential in Eq. (63) and the force in Eq. (64) we find

$$
\begin{array}{rl}
\nabla^{2} & K-\beta \kappa x \frac{\partial K}{\partial x}-\beta \kappa y \frac{\partial K}{\partial y}-\beta \kappa \eta z \frac{\partial K}{\partial z} \\
=-\varepsilon \beta^{2} \kappa^{2} a \eta z\left(1-\frac{x^{2}+y^{2}}{a^{2}}\right) .
\end{array}
$$

Inspection of the probabilistic representation of the solution and from the symmetry of the problem in the $(x, y)$ plane, now tells us that the solution must have the form

$$
K(\mathbf{x})=z\left[A+B\left(x^{2}+y^{2}\right)\right],
$$

this yields

$$
\begin{aligned}
4 B & -2 B \beta \kappa\left(x^{2}+y^{2}\right)-\beta \kappa \eta\left[A+B\left(x^{2}+y^{2}\right)\right] \\
& =-\varepsilon \beta^{2} \kappa^{2} a \eta\left(1-\frac{x^{2}+y^{2}}{a^{2}}\right),
\end{aligned}
$$

and comparing the coefficients of the polynomials now gives

$$
A=\frac{\varepsilon}{(\eta+2) a}\left[-4+\beta \kappa a^{2}(\eta+2)\right],
$$

$$
B=-\frac{\varepsilon \beta \kappa \eta}{(\eta+2) a},
$$

in agreement with the earlier calculation leading to Eq. (76) and thus the result given in Ref. [9].

Here, we also consider the perturbation to a force of type $\mathbf{F}_{\text {scat, } 2}$ derived in Eq. (30), which we write as

$$
\mathbf{F}^{\prime}=\mathbf{F}_{n}^{\prime}=\frac{\varepsilon^{\prime} \kappa}{a}\left[\mathbf{e}_{x} x z+\mathbf{e}_{y} y z-\mathbf{e}_{z} z^{2}\right],
$$

as it has no potential part. Here, we find

$$
\begin{gathered}
\nabla^{2} K^{\prime}-\beta \kappa x \frac{\partial K^{\prime}}{\partial x}-\beta \kappa y \frac{\partial K^{\prime}}{\partial y}-\beta \kappa \eta z \frac{\partial K^{\prime}}{\partial z} \\
=-\frac{\varepsilon^{\prime} \beta^{2} \kappa^{2}}{a} z\left(x^{2}+y^{2}-\eta z^{2}\right),
\end{gathered}
$$

where the ' on $K$ denotes that it is the form of $K$ for the perturbation $\mathbf{F}^{\prime}$. Here, we see that the solution must be of the form

$$
K^{\prime}(\mathbf{x})=z\left[A^{\prime}+B^{\prime}\left(x^{2}+y^{2}\right)+J^{\prime} z^{2}\right] .
$$

This gives

$$
\begin{gathered}
\left(6 J^{\prime}+4 B^{\prime}\right) z-2 \beta \kappa B^{\prime} z x^{2}-2 \beta \kappa B^{\prime} z y^{2} \\
-\beta \kappa \eta z\left(A^{\prime}+3 J^{\prime} z^{2}+B^{\prime} x^{2}+B^{\prime} y^{2}\right) \\
=-\frac{\varepsilon^{\prime} \beta^{2} \kappa^{2}}{a} z\left(x^{2}+y^{2}-\eta z^{2}\right),
\end{gathered}
$$

and thus,

$$
\begin{aligned}
A^{\prime} & =-\frac{2 \varepsilon^{\prime}}{a(2+\eta)}, \\
B^{\prime} & =\frac{\varepsilon^{\prime} \beta \kappa}{a(2+\eta)}, \\
J^{\prime} & =-\frac{\varepsilon^{\prime} \beta \kappa}{3 a} .
\end{aligned}
$$

This, therefore, gives a contribution to the steady state distribution,

$$
\frac{\Delta P_{s}(\mathbf{x})}{P_{0}(\mathbf{x})}=\frac{\beta \kappa \varepsilon^{\prime} z}{a}\left[\frac{1}{2+\eta}\left(x^{2}+y^{2}-\frac{2}{\beta \kappa}\right)-\frac{1}{3} z^{2}\right] .
$$

\section{B. Underdamped Brownian particles}

Here, the phase space corresponds to $\mathbf{Y}=(\mathbf{X}, \mathbf{V})$ where $\mathbf{X}$ and $\mathbf{V}$, respectively, denote the particle position and its velocity. Here, the Fokker-Planck operator is given by

$$
\begin{aligned}
H f(\mathbf{x}, \mathbf{v})= & -\nabla_{\mathbf{v}} \cdot\left[\frac{T \gamma}{m^{2}} \nabla_{\mathbf{v}} f+\frac{\gamma}{m} \mathbf{v} f-\frac{1}{m} \mathbf{F}_{t}(\mathbf{x}) f\right] \\
& +\nabla_{\mathbf{x}} \cdot[\mathbf{v} f] .
\end{aligned}
$$

where $\gamma$ is the friction coefficient, $m$ is the particle mass, and $\mathbf{F}_{t}$ is the total force on the particle, which is assumed to depend 
only on the position $\mathbf{x}$. For the unperturbed system, we take $\mathbf{F}_{t}(\mathbf{x})=-\nabla V(\mathbf{x})$. The equilibrium distribution is then given by

$$
P_{0}(\mathbf{x}, \mathbf{v})=\frac{1}{Z} \exp \left[-\frac{1}{2} \beta m \mathbf{v}^{2}-\beta V(\mathbf{x})\right] .
$$

In the perturbed system, we denote the additional force simply by $\mathbf{F}(\mathbf{x})$. This yields

$$
\frac{\Delta H P_{0}(\mathbf{x}, \mathbf{v})}{P_{0}(\mathbf{x}, \mathbf{v})}=-\beta \mathbf{v} \cdot \mathbf{F}(\mathbf{x})
$$

and, thus,

$$
\begin{aligned}
\Delta P_{0}(\mathbf{x}, \mathbf{v})= & -P_{0}(\mathbf{x}, \mathbf{v}) \beta \int_{0}^{\infty} d t\left[\left\langle\mathbf{V}_{t} \cdot \mathbf{F}\left(\mathbf{X}_{t}\right)\right\rangle_{\mathbf{x},-\mathbf{v}}\right. \\
& \left.-\langle\mathbf{V} \cdot \mathbf{F}(\mathbf{X})\rangle_{0}\right]
\end{aligned}
$$

where we have used $\mathbf{V}^{*}=-\mathbf{V}$. The result in Eq. (95) immediately gives us a physical interpretation of the modification of the steady state probability density function. We see that

$$
\int_{0}^{\infty} d t\left[\left\langle\mathbf{V}_{t} \cdot \mathbf{F}\left(\mathbf{X}_{t}\right)\right\rangle_{\mathbf{x},-\mathbf{v}}-\langle\mathbf{V} \cdot \mathbf{F}(\mathbf{X})\rangle_{0}\right]=\bar{W}(\mathbf{x},-\mathbf{v})-\bar{W}_{0},
$$

where $\bar{W}(\mathbf{x},-\mathbf{v})$ is the total work performed after a very long time $t^{*}$ by the nonconservative force on a particle started at the position $\mathbf{x}$ but and with initial velocity $-\mathbf{v}$, whereas $\bar{W}_{0}$ is the total work performed by the nonconservative force at the same late time $t^{*}$ for a particle whose initial velocity and position are taken from the unperturbed equilibrium probability density function. Both of these total works diverge as $t^{*} \rightarrow \infty$, but their difference is constant. When the perturbing force is purely conservative, i.e., $\mathbf{F}=-\nabla U$, we immediately obtain, much more transparently that in the Brownian case,

$$
\begin{aligned}
\Delta P_{0}(\mathbf{x}, \mathbf{v})= & P_{0}(\mathbf{x}, \mathbf{v}) \beta \int_{0}^{\infty} d t\left[\left\langle\mathbf{V}_{t} \cdot \nabla U\left(\mathbf{X}_{t}\right)\right\rangle_{\mathbf{x},-\mathbf{v}}\right. \\
& \left.-\langle\mathbf{V} \cdot \nabla U(\mathbf{X})\rangle_{0}\right],
\end{aligned}
$$

and as $\mathbf{V}=d \mathbf{X} / d t$ we find, using the same arguments as for the overdamped case,

$$
\Delta P_{0}(\mathbf{x}, \mathbf{v})=P_{0}(\mathbf{x}, \mathbf{v}) \beta\left[\langle U(\mathbf{X})\rangle_{0}-U(\mathbf{x})\right],
$$

thus recovering the corresponding modified Gibbs-Boltzmann distribution.

\section{Application to under generally damped particles in the optical trap model}

In principle, for a harmonic trap, all the average values in Eq. (95) can be computed. However, as for the overdamped case, the form of the solution suggests writing

$$
\Delta P_{0}(\mathbf{x}, \mathbf{v})=P_{0}(\mathbf{x}, \mathbf{v}) K(\mathbf{x}, \mathbf{v}) .
$$

This then yields

$$
\begin{aligned}
& \frac{T \gamma}{m^{2}} \nabla_{\mathbf{v}}^{2} K-\frac{\gamma}{m} \mathbf{v} \cdot \nabla_{\mathbf{v}} K-\mathbf{v} \cdot \nabla_{\mathbf{x}} K+\frac{1}{m} \nabla_{\mathbf{x}} V(\mathbf{x}) \cdot \nabla_{\mathbf{v}} K \\
& \quad=-\beta \mathbf{F} \cdot \mathbf{v} .
\end{aligned}
$$

There is a distinct advantage, at least, for complex perturbations by nonconservative forces, of using this algebraic formulation as opposed to the probabilistic representation. However, the probabilistic representation allows one to predict the polynomial form of $K$, and only the coefficients of this polynomial need be determined after substituting the correct form of $K$ into Eq. (100). This is because, in the expression $\left\langle\mathbf{V}_{t} \cdot \mathbf{F}\left(\mathbf{X}_{t}\right)\right\rangle_{\mathbf{x},-\mathbf{v}}$, we can see explicitly what terms in the initial position $\mathbf{x}$ and velocity $\mathbf{v}$ must arise by inspecting the form of $\mathbf{F}$ given in Eq. (64).

From the symmetry in the $(x, y)$ plane, the solution of Eq. (100) must have the form

$$
\begin{aligned}
K(\mathbf{x}, \mathbf{v})= & z\left[A+B\left(x^{2}+y^{2}\right)+C\left(x v_{x}+y v_{y}\right)+D\left(v_{x}^{2}+v_{y}^{2}\right)\right] \\
& +v_{z}\left[E+F\left(x^{2}+y^{2}\right)+G\left(x v_{x}+y v_{y}\right)\right. \\
& \left.+H\left(v_{x}^{2}+v_{y}^{2}\right)\right] .
\end{aligned}
$$

After simplification, the coefficients are given by

$$
\begin{aligned}
A= & \beta \varepsilon \kappa a-\frac{8 \varepsilon \gamma^{2}}{S}\left[6 \gamma^{2}+(8-5 \eta) \kappa m\right], \\
B= & -\frac{\eta \beta \varepsilon \kappa}{S}\left[(\eta-4)(\eta-2)(\kappa m)^{2}\right. \\
& \left.+4(2 \eta+1) \gamma^{2} \kappa m+12 \gamma^{4}\right], \\
C= & \frac{4 \eta \beta \varepsilon \gamma \kappa m}{S}\left[6 \gamma^{2}+(\eta+2) \kappa m\right], \\
D= & -\frac{2 \eta \beta \varepsilon \kappa m^{2}}{S}\left[6 \gamma^{2}+(4-\eta) \kappa m\right], \\
E= & \frac{8 \varepsilon \gamma m}{S}\left[6 \gamma^{2}+(4-\eta) \kappa m\right], \\
F= & -\frac{4 \beta \varepsilon \gamma \kappa m}{S}\left[6 \gamma^{2}+(\eta+2) \kappa m\right], \\
G= & -\frac{2 \beta \varepsilon \kappa m^{2}}{S}\left[6(\eta-2) \gamma^{2}+\eta(\eta-4) \kappa m\right], \\
H= & \frac{8 \beta \varepsilon \gamma \kappa m^{3}}{S}(\eta-1),
\end{aligned}
$$

where $S=a\left[\eta \kappa m+2 \gamma^{2}\right]\left[(\eta-4)^{2} \kappa m+6(2+\eta) \gamma^{2}\right]$. Using these expressions, we have thus computed the perturbed probability density function in the full $(\mathbf{x}, \mathbf{v})$ phase space. However, the final expressions are rather unwieldy, and we will therefore give explicit expressions for the marginal probability density functions of $\mathbf{X}$ and then $\mathbf{V}$.

Denoting the shift in the marginal steady state probability density function for the position by $\Delta P_{0}(\mathbf{x})=$ $\int d \mathbf{v} \Delta P_{0}(\mathbf{x}, \mathbf{v})=\int d \mathbf{v} K(\mathbf{x}, \mathbf{v}) P_{0}(\mathbf{x}, \mathbf{v})$, we find from Eq. (93)-(101),

$$
\begin{aligned}
\Delta & P_{0}(\mathbf{x}) \\
& =P_{0}(\mathbf{x}) \frac{\varepsilon z}{a}\left[\beta \kappa a^{2}-2+\eta\left(2-\beta \kappa \rho^{2}\right)\right. \\
& \left.\times \frac{(\eta-4)(\eta-2)(\kappa m)^{2}+4(2 \eta+1) \kappa m \gamma^{2}+12 \gamma^{4}}{\left[\eta \kappa m+2 \gamma^{2}\right]\left[(\eta-4)^{2} \kappa m+6(2+\eta) \gamma^{2}\right]}\right],
\end{aligned}
$$


where $\rho=\sqrt{x^{2}+y^{2}}$. In the underdamped limit $\gamma \rightarrow 0$, we find thus,

$$
\Delta P_{0}(\mathbf{x})=P_{0}(\mathbf{x}) \frac{\varepsilon z}{a}\left[\beta \kappa a^{2}-2+\frac{\eta-2}{\eta-4}\left(2-\beta \kappa \rho^{2}\right)\right],
$$

and in the overdamped limit $m \rightarrow 0$, we recover (for a third time) the result of Ref. [9] Eq. (76).

Using the modified probability density function in Eq. (110) can study various moments of the particle position. The average value of the height of the particle is given by

$$
\langle z\rangle=\frac{\varepsilon a}{\eta}\left(1-\frac{2}{\beta \kappa a^{2}}\right)
$$

whereas the average velocity in the direction $z$ is given by $\left\langle V_{z}\right\rangle=0$, after some mathematical cancellations which appear remarkable from a mathematical point of view but obvious from the simple physical insight that the particle is trapped! The above result for $\langle z\rangle$ is thus independent of the details of the dynamics (it is independent of the damping parameters). This surprising result is a consequence of the fact that the average velocity is zero (as obviously must be the case if the particle remains trapped): As the average velocity is zero, the mean position along the $z$ axis is given by a force balance equation where the friction term is zero, and, as the average thermal force and acceleration are zero, the average force balance equation depend only on the external forces acting on the particle and is thus independent of the damping parameters.

Here, we introduce the underlying frequency of the harmonic part of the trap $\omega_{0}^{2}=\kappa / m$ and the damping rate $\Gamma=$ $\gamma / m$. The quality factor is defined as $Q=\omega_{0} / \Gamma=\sqrt{\kappa m / \gamma^{2}}$, it quantifies the degree of damping in the system, large $Q$ corresponding to the underdamped regime and small $Q$ corresponding to the overdamped regime. In terms of these variables, which are often preferred in experimental studies, we find

$$
\begin{aligned}
\Delta P_{0}(\mathbf{x})= & P_{0}(\mathbf{x}) \frac{\varepsilon z}{a}\left[\beta \kappa a^{2}-2+\eta\left(2-\beta \kappa \rho^{2}\right)\right. \\
& \left.\times \frac{(\eta-4)(\eta-2) Q^{4}+4(2 \eta+1) Q^{2}+12}{\left[\eta Q^{2}+2\right]\left[(\eta-4)^{2} Q^{2}+6(2+\eta)\right]}\right] .
\end{aligned}
$$

Denoting the shift in the marginal steady state probability density function for the velocity by $\Delta P_{\mathrm{MB}}(\mathbf{v})=$ $\int d \mathbf{x} \Delta P_{0}(\mathbf{x}, \mathbf{v})=\int d \mathbf{x} K(\mathbf{x}, \mathbf{v}) P_{0}(\mathbf{x}, \mathbf{v})$, we find from Eqs. (93)-(101),

$$
\begin{aligned}
\Delta P_{\mathrm{MB}}(\mathbf{v})= & P_{\mathrm{MB}}(\mathbf{v}) \frac{8 \beta \kappa \varepsilon v_{z}}{a}\left(v_{\rho}^{2}-\frac{2}{\beta m}\right) \\
& \times \frac{\gamma \kappa m^{3}(\eta-1)}{\left[\eta \kappa m+2 \gamma^{2}\right]\left[(\eta-4)^{2} \kappa m+6(2+\eta) \gamma^{2}\right]},
\end{aligned}
$$

where $v_{\rho}=\sqrt{v_{x}^{2}+v_{y}^{2}}$ and

$$
P_{\mathrm{MB}}(\mathbf{v})=\left(\frac{\beta m}{2 \pi}\right)^{3 / 2} \exp \left(-\frac{\beta m \mathbf{v}^{2}}{2}\right)
$$

is the Maxwell-Boltzmann velocity distribution. Interestingly, the marginal probability distributions for $v_{x}, v_{y}$, and $v_{z}$ do not change in first order perturbation theory and are thus given by the Maxwell distribution. The important point to be made here is that the effects of the nonconservative force feed through into the velocity distribution so that even the fundamental Maxwell-Boltzmann distribution is modified by nonconservative forces. This important result is experimentally verified in the accompanying paper [11], confirming the experimental relevance of the theoretical study carried out here.

In Ref. [9], the steady state current was computed for an overdamped Brownian particle. Here, we have currents in both position and velocity spaces. From Eq. (92), we see that currents $\mathbf{J}_{\mathbf{x}}$ and $\mathbf{J}_{\mathbf{v}}$ can be read off from

$$
\begin{aligned}
\frac{\partial P(\mathbf{x}, \mathbf{v}, t)}{\partial t}= & -H P(\mathbf{x}, \mathbf{v}, t)=-\nabla_{\mathbf{x}} \cdot \mathbf{J}_{\mathbf{x}}(\mathbf{x}, \mathbf{v}, t) \\
& -\nabla_{\mathbf{v}} \cdot \mathbf{J}_{\mathbf{v}}(\mathbf{x}, \mathbf{v}, t)
\end{aligned}
$$

where

$$
\begin{aligned}
\mathbf{J}_{\mathbf{x}}(\mathbf{x}, \mathbf{v}, t)= & \mathbf{v} P(\mathbf{x}, \mathbf{v}, t), \\
\mathbf{J}_{\mathbf{v}}(\mathbf{x}, \mathbf{v}, t)= & -\frac{T \gamma}{m^{2}} \nabla_{\mathbf{v}} P(\mathbf{x}, \mathbf{v}, t)-\frac{\gamma}{m} \mathbf{v} P(\mathbf{x}, \mathbf{v}, t) \\
& +\frac{1}{m} \mathbf{F}_{t}(\mathbf{x}) P(\mathbf{x}, \mathbf{v}, t) .
\end{aligned}
$$

The effective currents in position space $\overline{\mathbf{J}_{\mathbf{x}}}(\mathbf{x})$ and velocity space $\overline{\mathbf{J}_{\mathbf{v}}}(\mathbf{v})$ can be found by integrating Eq. (116) over $\mathbf{v}$ and $\mathbf{x}$, respectively, to obtain the Fokker-Plank equation for the marginal probability distribution in position $\mathbf{x}$ and velocity $\mathbf{v}$, respectively. From this, and using the divergence theorem, the associated currents can be read off as

$$
\begin{aligned}
\overline{\mathbf{J}}_{\mathbf{x}}(\mathbf{x}, t)= & \int d \mathbf{v} \mathbf{v} P(\mathbf{x}, \mathbf{v}, t) \\
\overline{\mathbf{J}_{\mathbf{v}}}(\mathbf{v}, t)= & -\int d \mathbf{x}\left[\frac{T \gamma}{m^{2}} \nabla_{\mathbf{v}} P(\mathbf{x}, \mathbf{v}, t)+\frac{\gamma}{m} \mathbf{v} P(\mathbf{x}, \mathbf{v}, t)\right. \\
& \left.-\frac{1}{m} \mathbf{F}_{t}(\mathbf{x}) P(\mathbf{x}, \mathbf{v}, t)\right] .
\end{aligned}
$$

In the nonequilibrium steady state, we find that the currents are given, to first order in the perturbation by the nonconservative force as

$$
\begin{aligned}
\overline{\mathbf{J}_{\mathbf{x}}}(\mathbf{x})= & \int d \mathbf{v} \mathbf{v} \Delta P_{0}(\mathbf{x}, \mathbf{v}) \\
\overline{\mathbf{J}_{\mathbf{v}}}(\mathbf{v})= & -\int d \mathbf{x}\left[\frac{T \gamma}{m^{2}} \nabla_{\mathbf{v}} \Delta P_{0}(\mathbf{x}, \mathbf{v})+\frac{\gamma}{m} \mathbf{v} \Delta P_{0}(\mathbf{x}, \mathbf{v})\right. \\
& \left.-\frac{1}{m} \mathbf{F}(\mathbf{x}) P_{0}(\mathbf{x}, \mathbf{v})+\frac{1}{m} \nabla V(\mathbf{x}) \Delta P_{0}(\mathbf{x}, \mathbf{v})\right]
\end{aligned}
$$

These expressions can then be rewritten in terms of the function $K(\mathbf{x}, \mathbf{v})$ to give

$$
\begin{aligned}
\overline{\mathbf{J}_{\mathbf{x}}}(\mathbf{x})= & \int d \mathbf{v} \mathbf{v} P_{0}(\mathbf{x}, \mathbf{v}) K(\mathbf{x}, \mathbf{v}) \\
\overline{\mathbf{J}_{\mathbf{v}}}(\mathbf{v})= & -\int d \mathbf{x}\left[\frac{T \gamma}{m^{2}} P_{0}(\mathbf{x}, \mathbf{v}) \nabla_{\mathbf{v}} K(\mathbf{x}, \mathbf{v})-\frac{1}{m} \mathbf{F}(\mathbf{x}) P_{0}(\mathbf{x}, \mathbf{v})\right. \\
& \left.+\frac{1}{m} \nabla V(\mathbf{x}) P_{0}(\mathbf{x}, \mathbf{v}) K(\mathbf{x}, \mathbf{v})\right]
\end{aligned}
$$



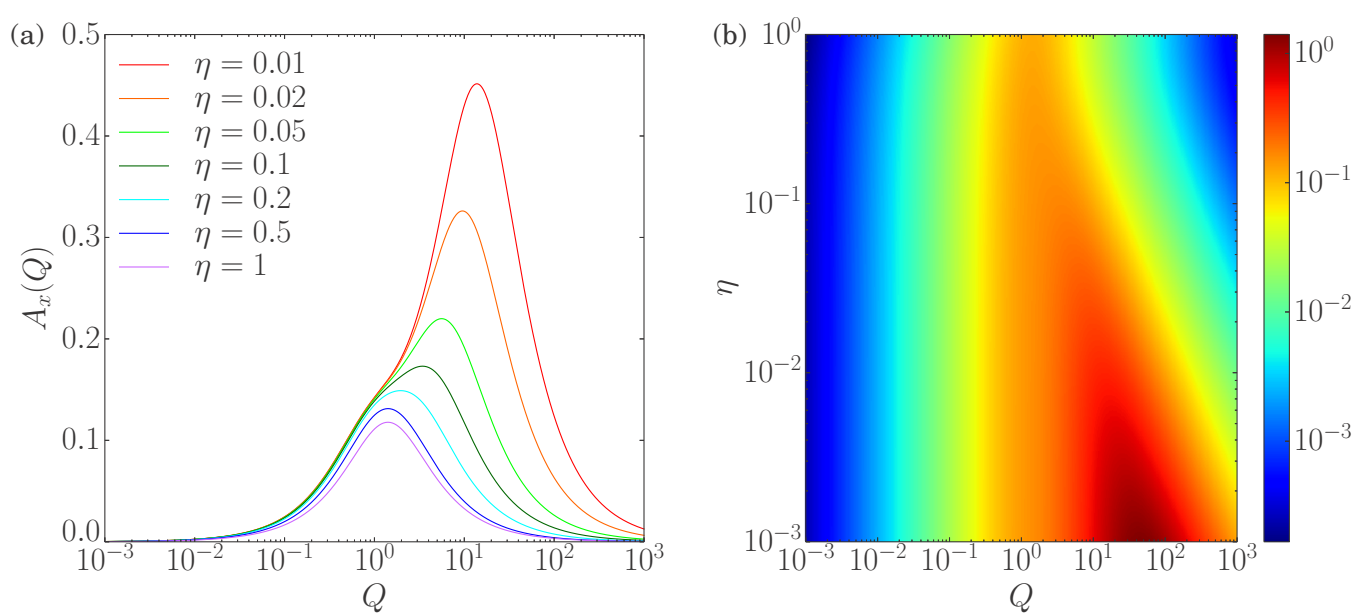

FIG. 1. (a) Behavior of the spatial current amplitude $A_{x}(\eta, Q)$ as given by Eq. (129) for various values of $\eta$. This amplitude has a single maximum as a function of $Q$, and the nonequilibrium spatial current can therefore be maximized by tuning $Q$. (b) Two dimensional intensity plot of $A(\eta, Q)$.

Using the form of $K(\mathbf{x}, \mathbf{v})$ derived for the optical trap model, we obtain

$$
\begin{aligned}
\overline{\mathbf{J}_{\mathbf{x}}}(\mathbf{x})= & P_{0}(\mathbf{x}) \frac{4 \varepsilon \kappa \gamma}{a} \frac{6 \gamma^{2}+(\eta+2) \kappa m}{\left[\eta \kappa m+2 \gamma^{2}\right]\left[(\eta-4)^{2} \kappa m+6(2+\eta) \gamma^{2}\right]} \\
& \times\left[\eta z \rho \mathbf{e}_{\rho}+\left(\frac{2}{\beta \kappa}-\rho^{2}\right) \mathbf{e}_{\mathbf{z}}\right],
\end{aligned}
$$

where $\mathbf{e}_{\rho}$ is the radial unit vector in polar coordinates (recall here $\rho=\sqrt{x^{2}+y^{2}}$ ). In the limit $m \rightarrow 0$, we recover the result of Ref. [9] for the overdamped case,

$$
\overline{\mathbf{J}_{\mathbf{x}}}(\mathbf{x})=P_{0}(\mathbf{x}) \frac{2 \varepsilon \beta \kappa D}{a(2+\eta)}\left[\eta z \rho \mathbf{e}_{\rho}+\left(\frac{2}{\beta \kappa}-\rho^{2}\right) \mathbf{e}_{\mathbf{z}}\right],
$$

where $D$ is the effective diffusion constant as defined by the Einstein relation $D=T / \gamma$. In the underdamped limit $\gamma \rightarrow 0$, we obtain

$$
\overline{\mathbf{J}_{\mathbf{x}}}(\mathbf{x})=P_{0}(\mathbf{x}) \frac{4 \varepsilon \gamma}{a m} \frac{\eta+2}{\eta(\eta-4)^{2}}\left[\eta z \rho \mathbf{e}_{\rho}+\left(\frac{2}{\beta \kappa}-\rho^{2}\right) \mathbf{e}_{\mathbf{z}}\right] .
$$

Remarkably, the geometry of the current field $\overline{\mathbf{J}_{\mathbf{x}}}(\mathbf{x})$ in space is always the same; the damping parameters only appear via an overall space-independent amplitude term $A_{x}(\eta, Q)$ such that

$$
\overline{\mathbf{J}_{\mathbf{x}}}(\mathbf{x})=P_{0}(\mathbf{x}) \frac{4 \varepsilon \omega_{0}}{a} A_{x}(\eta, Q)\left[\eta z \rho \mathbf{e}_{\rho}+\left(\rho_{c}^{2}-\rho^{2}\right) \mathbf{e}_{\mathbf{z}}\right],
$$

where $\rho_{c}=\sqrt{2 / \beta \kappa}$ and

$$
A_{x}(\eta, Q)=\frac{Q\left[(\eta+2) Q^{2}+6\right]}{\left[\eta Q^{2}+2\right]\left[(\eta-4)^{2} Q^{2}+6(2+\eta)\right]} .
$$

We see above that when $Q \rightarrow 0$ and $Q \rightarrow \infty, A_{x} \rightarrow 0$ and thus the amplitude of the nonequilibrium current can be maximized at a given value of $Q$ when all other physical parameters are fixed as can be clearly seen in Fig. 1(a). We also see that the amplitude increases as $\eta$ decreases and this is to be expected as the trapping in the $z$ direction becomes weaker on reducing $\eta$.
As pointed out in Ref. [9], at the point $\rho_{c}$ the direction of the current in the $z$ direction reverses, the value of $\rho_{c}$ is the value where the particles potential energy due to the harmonic trap attains the equilibrium value as predicted by the equipartition of energy. We also see that the current in the $\rho$ direction is positive for $z>0$ and negative for $z<0$. The current in the radial (polar coordinate) direction $\rho$ is in the direction $\mathbf{e}_{\rho}$ and its sign is proportional to $z$, thus the current flows away from the origin on the $(x, y)$ plane when $z>0$ and towards the origin when $z<0$.

The current in velocity space is given by $\mathbf{v}$,

$$
\begin{aligned}
\overline{\mathbf{J}_{\mathbf{v}}}(\mathbf{v})= & -P_{\mathrm{MB}}(\mathbf{v}) \frac{2 \varepsilon \kappa m}{a} \\
& \times \frac{\eta(4-\eta) \kappa m+2(\eta+2) \gamma^{2}}{\left[\eta \kappa m+2 \gamma^{2}\right]\left[(\eta-4)^{2} \kappa m+6(2+\eta) \gamma^{2}\right]} \\
& \times\left[v_{z}\left(v_{x} \mathbf{e}_{\mathbf{x}}+v_{y} \mathbf{e}_{\mathbf{y}}\right)+\left(\frac{2}{\beta m}-v_{\rho}^{2}\right) \mathbf{e}_{\mathbf{z}}\right],
\end{aligned}
$$

where $v_{\rho}=\sqrt{v_{x}^{2}+v_{y}^{2}}$. In the overdamped limit $m \rightarrow 0$, one finds

$$
\overline{\mathbf{J}_{\mathbf{v}}}(\mathbf{v})=-P_{\mathrm{MB}}(\mathbf{v}) \frac{\varepsilon \kappa m}{3 a \gamma^{2}}\left[v_{z}\left(v_{x} \mathbf{e}_{\mathbf{x}}+v_{y} \mathbf{e}_{\mathbf{y}}\right)+\left(\frac{2}{\beta m}-v_{\rho}^{2}\right) \mathbf{e}_{\mathbf{z}}\right],
$$

and the limit $\gamma \rightarrow 0$, we find

$$
\begin{aligned}
\overline{\mathbf{J}_{\mathbf{v}}}(\mathbf{v})= & -P_{\mathrm{MB}}(\mathbf{v}) \frac{2 \varepsilon}{a(4-\eta)}\left[v_{z}\left(v_{x} \mathbf{e}_{\mathbf{x}}+v_{y} \mathbf{e}_{\mathbf{y}}\right)\right. \\
& \left.+\left(\frac{2}{\beta m}-v_{\rho}^{2}\right) \mathbf{e}_{\mathbf{z}}\right]
\end{aligned}
$$

Again, we see that the geometric structure in phase space is independent of the damping parameters and that the damping parameters only appear in a velocity-independent constant amplitude. Remarkably, the geometric structure is basically 

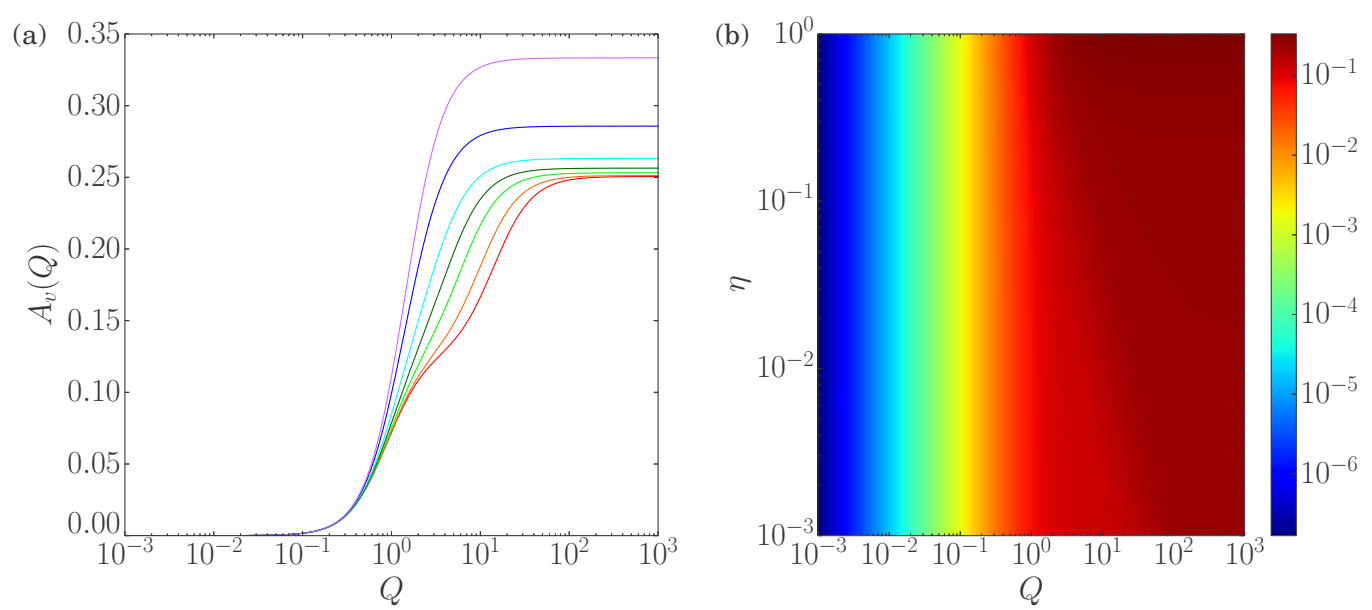

FIG. 2. (a) Behavior of $A_{v}(\eta, Q)$, the amplitude of the current in velocity space, for several values of $\eta$. (b) Intensity plot of $A_{v}(\eta, Q)$.

identical to that of the position current if we reverse the direction and make the correspondence $\mathbf{v} \equiv \mathbf{x}$. The geometry of the current is also only dependent on the temperature and particle mass and is independent of the parameters of the trap. Here, the $Q$-dependent amplitude of the current $A_{v}(\eta, Q)$ is defined such that

$$
\begin{aligned}
\overline{\mathbf{J}_{\mathbf{v}}}(\mathbf{v})= & -P_{\mathrm{MB}}(\mathbf{v}) \frac{2 \varepsilon}{a} A_{v}(\eta, Q)\left[v_{z}\left(v_{x} \mathbf{e}_{\mathbf{x}}+v_{y} \mathbf{e}_{\mathbf{y}}\right)\right. \\
& \left.+\left(v_{c}^{2}-v_{\rho}^{2}\right) \mathbf{e}_{\mathbf{z}}\right],
\end{aligned}
$$

where $v_{c}=\sqrt{2 / \beta m}$ and

$$
A_{v}(\eta, Q)=\frac{Q^{2}\left[\eta(4-\eta) Q^{2}+2(\eta+2)\right]}{\left[\eta Q^{2}+2\right]\left[(\eta-4)^{2} Q^{2}+6(2+\eta)\right]} .
$$

Here, we find that, as $Q \rightarrow 0, A_{v}(\eta, Q) \rightarrow 0$, whereas $A_{v}(\eta, Q)=1 /(4-\eta)$ as $Q \rightarrow \infty$. This function is monotonically increasing with $Q$ as can be seen in Fig. 2(a), and it reaches a plateau at large $Q$. In contrast to the amplitude of the spatial current $A_{x}(\eta, Q)$, we see that the amplitude is largest for large values of $\eta$, i.e., for strong trapping in the $z$ direction.

Note that the apparent divergence for $\eta=4$ is removed due to the presence of terms of next order in $\gamma$, however, the point $\eta=4$ should present some form of resonance in the current. The value $\eta=4$ is, however, far from its typical experimental value which is around $0.3[11]$.

\section{Application to general particles in the optical trap model with the $F_{\text {scat, } 2}$ force}

We want to find $\Delta P_{s}(\mathbf{x}, \mathbf{v})=P_{0}(\mathbf{x}, \mathbf{v}) K^{\prime}(\mathbf{x}, \mathbf{v})$ satisfying Eq. (100) with $\mathbf{F}^{\prime}$ defined in Eq. (84) instead of $\mathbf{F}$. The solution of this equation must have the form

$$
\begin{aligned}
K^{\prime}(\mathbf{x}, \mathbf{v})= & z\left[A^{\prime}+B^{\prime}\left(x^{2}+y^{2}\right)+C^{\prime}\left(x v_{x}+y v_{y}\right)\right. \\
& \left.+D^{\prime}\left(v_{x}^{2}+v_{y}^{2}\right)\right]+v_{z}\left[E^{\prime}+F^{\prime}\left(x^{2}+y^{2}\right)\right. \\
& \left.+G^{\prime}\left(x v_{x}+y v_{y}\right)+H^{\prime}\left(v_{x}^{2}+v_{y}^{2}\right)\right] \\
& +J^{\prime} z^{3}+K^{\prime} z^{2} v_{z}+L^{\prime} z v_{z}^{2}+M^{\prime} v_{z}^{3} .
\end{aligned}
$$

After simplification, the coefficients are given by

$$
\begin{aligned}
A^{\prime} & =-\frac{4 \varepsilon^{\prime} \gamma^{2}}{S}\left[6 \gamma^{2}+(8-5 \eta) \kappa m\right], \\
B^{\prime} & =\frac{\beta \varepsilon^{\prime} \kappa}{S}\left[\eta(4-\eta)(\kappa m)^{2}+4(4-\eta) \gamma^{2} \kappa m+12 \gamma^{4}\right], \\
C^{\prime} & =\frac{2 \eta \beta \varepsilon^{\prime} \gamma \kappa m}{S}\left[6 \gamma^{2}+(\eta+2) \kappa m\right], \\
D^{\prime} & =-\frac{\eta \beta \varepsilon^{\prime} \kappa m^{2}}{S}\left[6 \gamma^{2}+(4-\eta) \kappa m\right], \\
E^{\prime} & =\frac{4 \varepsilon^{\prime} \gamma m}{S}\left[6 \gamma^{2}+(4-\eta) \kappa m\right], \\
F^{\prime} & =-\frac{2 \beta \varepsilon^{\prime} \gamma \kappa m}{S}\left[6 \gamma^{2}+(\eta+2) \kappa m\right], \\
G^{\prime} & =-\frac{\beta \varepsilon^{\prime} \kappa m^{2}}{S}\left[6(\eta-2) \gamma^{2}+\eta(\eta-4) \kappa m\right], \\
H^{\prime} & =\frac{4 \beta \varepsilon \gamma \kappa m^{3}}{S}(\eta-1), \\
J^{\prime} & =-\frac{\beta \epsilon^{\prime} \kappa}{3 a}, \\
K^{\prime} & =L^{\prime}=M^{\prime}=0,
\end{aligned}
$$

where $S=a\left[\eta \kappa m+2 \gamma^{2}\right]\left[(\eta-4)^{2} \kappa m+6(2+\eta) \gamma^{2}\right]$. Denoting the change due to the nonconservative force in marginal steady state probability density function for the position by $\Delta P_{s}(\mathbf{x})=\int d \mathbf{v} K^{\prime}(\mathbf{x}, \mathbf{v}) P_{0}(\mathbf{x}, \mathbf{v})$, we find from Eqs. (93) and (135),

$$
\Delta P_{s}(\mathbf{x})=P_{0}(\mathbf{x}) \frac{\beta \kappa \varepsilon^{\prime} z}{a}\left[\left(x^{2}+y^{2}-\frac{2}{\beta \kappa}\right) \frac{\eta(4-\eta)(\kappa m)^{2}+4(4-\eta) \kappa m \gamma^{2}+12 \gamma^{4}}{\left[\eta \kappa m+2 \gamma^{2}\right]\left[(\eta-4)^{2} \kappa m+6(2+\eta) \gamma^{2}\right]}-\frac{z^{2}}{3}\right] .
$$


We recover the Eq. (91) taking the limit $m \rightarrow 0$. Denoting the marginal steady state probability density function for the velocity by $\Delta P_{\mathrm{MB}}^{\prime}(\mathbf{v})=\int d \mathbf{x} K^{\prime}(\mathbf{x}, \mathbf{v}) P_{0}(\mathbf{x}, \mathbf{v})$, we find from Eqs. (93) and (135),

$$
\Delta P_{\mathrm{MB}}^{\prime}(\mathbf{v})=P_{\mathrm{MB}}(\mathbf{v}) \frac{4 \beta \kappa \varepsilon^{\prime} v_{z}}{a}\left(v_{x}^{2}+v_{y}^{2}-\frac{2}{\beta m}\right) \frac{\gamma \kappa m^{3}(\eta-1)}{\left[\eta \kappa m+2 \gamma^{2}\right]\left[(\eta-4)^{2} \kappa m+6(2+\eta) \gamma^{2}\right]} .
$$

The effective current in position space is defined as $\overline{\mathbf{J}_{\mathbf{x}}^{\prime}}(\mathbf{x})=\int d \mathbf{v} \mathbf{v} P_{0}(\mathbf{x}, \mathbf{v}) K^{\prime}(\mathbf{x}, \mathbf{v})$ from Eq. (123). Using the expression of $K^{\prime}$ from Eq. (135), we get

$$
\overline{\mathbf{J}_{\mathbf{x}}^{\prime}}(\mathbf{x})=P_{0}(\mathbf{x}) \frac{2 \kappa \gamma \varepsilon^{\prime}}{a} \frac{6 \gamma^{2}+(\eta+2) \kappa m}{\left[\eta \kappa m+2 \gamma^{2}\right]\left[(\eta-4)^{2} \kappa m+6(2+\eta) \gamma^{2}\right]}\left[\eta z\left(x \mathbf{e}_{\mathbf{x}}+y \mathbf{e}_{\mathbf{y}}\right)-\left(x^{2}+y^{2}-\frac{2}{\beta \kappa}\right) \mathbf{e}_{\mathbf{z}}\right] .
$$

The effective current in velocity space is defined from Eq. (124) as

$$
\overline{\mathbf{J}_{\mathbf{v}}^{\prime}}(\mathbf{v})=-\int d \mathbf{x}\left[\frac{T \gamma}{m^{2}} P_{0}(\mathbf{x}, \mathbf{v}) \nabla_{\mathbf{v}} K^{\prime}(\mathbf{x}, \mathbf{v})-\frac{1}{m} \mathbf{F}^{\prime}(\mathbf{x}) P_{0}(\mathbf{x}, \mathbf{v})+\frac{1}{m} \nabla V(\mathbf{x}) P_{0}(\mathbf{x}, \mathbf{v}) K^{\prime}(\mathbf{x}, \mathbf{v})\right] .
$$

After some simplifications, we find

$$
\overline{\mathbf{J}_{\mathbf{v}}^{\prime}}(\mathbf{v})=-P_{\mathrm{MB}}(\mathbf{v}) \frac{\varepsilon^{\prime} \kappa m}{a} \frac{\eta(4-\eta) \kappa m+2(\eta+2) \gamma^{2}}{\left[\eta \kappa m+2 \gamma^{2}\right]\left[(\eta-4)^{2} \kappa m+6(2+\eta) \gamma^{2}\right]}\left[v_{z}\left(v_{x} \mathbf{e}_{\mathbf{x}}+v_{y} \mathbf{e}_{\mathbf{y}}\right)-\left(v_{x}^{2}+v_{y}^{2}-\frac{2}{\beta m}\right) \mathbf{e}_{\mathbf{z}}\right] .
$$

We see that the above results currents induced by the force $\mathbf{F}^{\prime}$ are remarkably similar to those induced by the nonconservative part of the force $\mathbf{F}_{n}$ of the force $\mathbf{F}^{\prime}$. This can, in fact, be seen much more easily by noting that the nonconservative parts of $\mathbf{F}$ and $\mathbf{F}^{\prime}$ are proportional. Indeed, we can write

$$
\begin{aligned}
\frac{\mathbf{F}^{\prime}}{\varepsilon^{\prime}} & =\frac{\kappa}{a}\left(\nabla\left[\frac{x^{2}+y^{2}}{2}-\frac{z^{3}}{3}\right]-\mathbf{e}_{z} \frac{x^{2}+y^{2}}{2}\right) \\
& =-\nabla U+\frac{\mathbf{F}}{2 \varepsilon}
\end{aligned}
$$

where $U=-\kappa\left(\frac{x^{2}+y^{2}}{2}-\frac{z^{3}}{3}\right)$ is a potential difference between the two nonconservative forces. This potential term does not contribute to the current at the order if perturbation theory used here, thus, explaining the simple relationship between the two currents found here.

\section{E. Comparison with numerical simulation}

We will now verify the analytical results for the nonequilibrium steady state probability distribution function and the associated currents, presented above, by comparing them with the results of the numerical integration of the corresponding Langevin equations, which are given by

$$
\begin{aligned}
m \ddot{x}+\gamma \dot{x}+\kappa_{x} x & =\sqrt{2 \gamma T} \xi_{x}, \\
m \ddot{y}+\gamma \dot{y}+\kappa_{y} y & =\sqrt{2 \gamma T} \xi_{y}, \\
m \ddot{z}+\gamma \dot{z}+\kappa_{z} z & =\sqrt{2 \gamma T} \xi_{z}+\varepsilon \kappa_{x} a\left(1-\frac{x^{2}+y^{2}}{a^{2}}\right) .
\end{aligned}
$$

Here, $\xi_{x}, \xi_{y}$, and $\xi_{z}$ are independent Gaussian white noises. We consider the system with the following adimensionalized parameters, where $a=1$ and $\kappa_{x} / m=\omega_{0}^{2}=1$ such that:

$$
\begin{aligned}
\ddot{x}+Q^{-1} \dot{x}+x & =\sqrt{2 Q^{-1}} \sigma \xi_{x}, \\
\ddot{y}+Q^{-1} \dot{y}+\eta_{y} y & =\sqrt{2 Q^{-1}} \sigma \xi_{y}, \\
\ddot{z}+Q^{-1} \dot{z}+\eta_{z} z & =\sqrt{2 Q^{-1}} \sigma \xi_{z}+\varepsilon\left(1-x^{2}-y^{2}\right),
\end{aligned}
$$

where $Q=\omega_{0} / \Gamma$ is the quality factor, $\sigma^{2}=\left(\beta \kappa_{x} a^{2}\right)^{-1}, \eta_{y}=$ $\kappa_{y} / \kappa_{x}$, and $\eta_{z}=\kappa_{z} / \kappa_{x}$. The numerical integration of these equations is carried out using the algorithm of Sivak et al. [21]. The probability density function is estimated using a spatial binning procedure for boxes of volume $v=\Delta x \Delta y \Delta z$ as

$$
P_{0}(\mathbf{x})=\frac{1}{v \mathcal{N}} \sum_{i=1}^{\mathcal{N}} I\left(\mathbf{X}_{t_{i}}, \mathbf{x}\right),
$$

where $I\left(\mathbf{X}_{t_{i}}, \mathbf{x}\right)=1$ if $\mathbf{X}_{t_{i}}$, the particle position at time $t_{i}=$ $i \Delta t$, is in the bin of $\mathbf{x}$ and $\mathcal{N}$ is the total number of points in the trajectory. The spatial current is computed from Eq. (119) using the estimator,

$$
\overline{\mathbf{J}_{\mathbf{x}}}(\mathbf{x})=\frac{1}{v \mathcal{N}} \sum_{i=1}^{\mathcal{N}} I\left(\mathbf{X}_{t_{i}}, \mathbf{x}\right) \mathbf{V}_{t_{i}},
$$

where $\mathbf{V}_{t_{i}}$ is the measured particle velocity at time $t_{i}$.

The numerically obtained value of the steady state probability distribution for the parameters $\varepsilon=0.1, \sigma=0.5, \eta_{y}=$ $1, \eta_{z}=0.2$, and $Q=1$ whereas the bins are taken with respect to $\Delta x=\Delta y=6 \sigma / 100$ and $\Delta z=6 \sigma_{z} / 100$ (where $\sigma_{z}=$ $\sigma / \sqrt{\eta})$. The simulation is carried out using for the integration time step $\Delta t=10^{-2}$ and over a total measurement time $t_{m}=$ 10 for a total of $\mathcal{N}=5.10^{9}$ different trajectories. We show in Fig. 3(a) the estimated steady state distribution as a grayscale plot along with the vector field plot of the current (shown as arrows). In Fig. 3(b), we see the corresponding quantities predicted by the perturbation theory employed in this paper. The agreement is excellent as it should be for the small value of $\varepsilon$ used here.

\section{POWER SPECTRA FOR UNDERDAMPED PARTICLES IN PERTURBED HARMONIC POTENTIALS}

The computation of the spectral density function for the particle position in the trap model considered here has been carried out in Ref. [10]. Here, we generalize this calculation to the case of underdamped Brownian particles and show that 

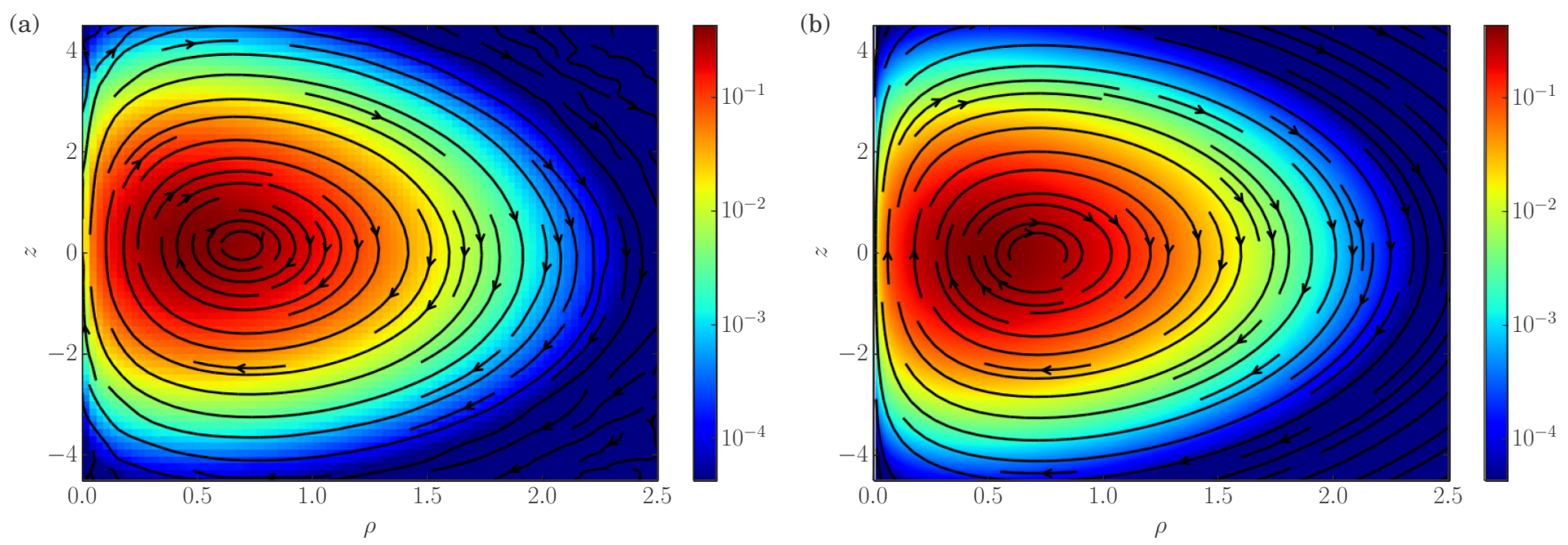

FIG. 3. (a) Numerical simulation results for the stationary probability density $P_{0}$ obtained from Eqs. (155) $-(157)$ with $\varepsilon=0.1, \sigma=$ $0.5, \eta_{y}=1, \eta_{z}=0.2$, and $Q=1$ obtained from the estimator given by Eq. (158). The vector field corresponds to the local stochastic average of the local velocity $\mathbf{V}$ to obtain the stationary current $\overline{\mathbf{J}_{\mathbf{x}}}(\rho, z)$ via Eq. (159). (b) For the same parameters, the leading order expressions of the probability density and of the current are plotted here from Eqs. (93) and (128), respectively.

significant differences arise. We then verify the calculation by comparing it with numerical simulations.

\section{A. Analytical calculation}

Here, we compute the power function of an underlying harmonic process subjected to a perturbing nonconservative force. The equations of motion for the components of the particle position are again given by Eqs. (152)-(154), and, in this particular approximation, only the steady state distribution of $z$ is changed (the positions $x$ and $y$ are not coupled to the nonconservative force, and the process $z$ is a slave to the positions $(x, y)$ [22]). The addition of the perturbation gives $z$ a nonzero average value given by

$$
\langle z\rangle=\frac{\varepsilon a}{\eta}\left\langle 1-\frac{x^{2}+y^{2}}{a^{2}}\right\rangle=\frac{\varepsilon a}{\eta}\left(1-\frac{2 T}{\kappa a^{2}}\right) .
$$

Due to the linearity of Eq. (154), we can decompose $z$ as

$$
z(t)=\langle z\rangle+\zeta_{e f}(t)+\zeta_{n}(t),
$$

where $z_{e f}$ is the equilibrium fluctuation due to the thermal noise obeying

$$
m \ddot{\zeta}_{e f}+\gamma \dot{\zeta}_{e f}+\kappa \eta \zeta_{e f}=\sqrt{2 \gamma T} \xi_{z},
$$

and $\zeta_{n}$ is the fluctuation in $z$ due to the fluctuations of the nonconservative force; $\zeta_{n}$ then obeys

$$
m \ddot{\zeta}_{n}+\gamma \dot{\zeta}_{n}+\kappa \eta \zeta_{n}=f_{n},
$$

where

$$
f_{n}=-\frac{\varepsilon \kappa}{a}\left(x^{2}-\left\langle x^{2}\right\rangle+y^{2}-\left\langle y^{2}\right\rangle\right)
$$

is the fluctuating part of the nonconservative noise in the $z$ direction. Fourier transforming in time then gives

$$
\tilde{\zeta}_{e f}(\omega)=\frac{\sqrt{2 T \gamma} \tilde{\xi}_{z}(\omega)}{-m \omega^{2}+i \omega \gamma+\kappa \eta},
$$

and

$$
\tilde{\zeta}_{n}(\omega)=\frac{f_{n}(\omega)}{-m \omega^{2}+i \omega \gamma+\kappa \eta} .
$$

The power spectrum of the fluctuations $\zeta=\zeta_{e f}+\zeta_{n}$ is then given by

$$
\left\langle\tilde{\zeta}(\omega) \tilde{\zeta}\left(\omega^{\prime}\right)\right\rangle=\left\langle\tilde{\zeta}_{e f}(\omega) \tilde{\zeta}_{e f}\left(\omega^{\prime}\right)\right\rangle+\left\langle\tilde{\zeta}_{n}(\omega) \tilde{\zeta}_{n}\left(\omega^{\prime}\right)\right\rangle,
$$

where

$$
\left\langle\tilde{\zeta}_{e f}(\omega) \tilde{\zeta}_{e f}\left(\omega^{\prime}\right)\right\rangle=2 \pi \delta\left(\omega+\omega^{\prime}\right) S_{z z-\mathrm{eq}}(\omega)
$$

is just given by the ordinary equilibrium fluctuation spectrum,

$$
S_{z z-\mathrm{eq}}(\omega)=\frac{2 T \gamma}{\left(\kappa \eta-m \omega^{2}\right)^{2}+\omega^{2} \gamma^{2}} .
$$

The contribution from the nonconservative force to the power spectrum is

$$
\left\langle\tilde{\zeta}_{n}(\omega) \tilde{\zeta}_{n}\left(\omega^{\prime}\right)\right\rangle=2 \pi \delta\left(\omega+\omega^{\prime}\right) S_{n n}(\omega) .
$$

Defining

$$
\left\langle\tilde{f}_{n}(\omega) \tilde{f}_{n}\left(\omega^{\prime}\right)\right\rangle=2 \pi \delta\left(\omega+\omega^{\prime}\right) C(\omega),
$$

then gives

$$
S_{n n}(\omega)=\frac{C(\omega)}{\left(\kappa \eta-m \omega^{2}\right)^{2}+\omega^{2} \gamma^{2}} .
$$

To proceed with the computation of $C(\omega)$, we use

$$
\widetilde{x^{2}}(\omega)=\frac{1}{2 \pi} \int d \nu \tilde{x}(\omega-v) \tilde{x}(\nu),
$$

and so the associated connected correlation function is given by

$$
\begin{aligned}
\left\langle\tilde{x^{2}}(\omega) \tilde{x^{2}}\left(\omega^{\prime}\right)\right\rangle_{c}= & \frac{1}{(2 \pi)^{2}} \int d \nu d v^{\prime}\{\langle\tilde{x}(\omega-v) \\
& \left.\times \tilde{x}\left(\omega^{\prime}-v^{\prime}\right)\right\rangle\left\langle\tilde{x}(v) \tilde{x}\left(v^{\prime}\right)\right\rangle+\langle\tilde{x}(\omega-v) \\
& \left.\left.\times \tilde{x}\left(v^{\prime}\right)\right\rangle\left\langle\tilde{x}\left(\omega^{\prime}-v^{\prime}\right) \tilde{x}(v)\right\rangle\right\}
\end{aligned}
$$


In terms of the equilibrium spectral function for $x$,

$$
S_{x x-\mathrm{eq}}(\omega)=\frac{2 T \gamma}{\left(\kappa-m \omega^{2}\right)^{2}+\omega^{2} \gamma^{2}},
$$

we thus find

$$
\begin{aligned}
& \left\langle\widetilde{x^{2}}(\omega) \widetilde{x^{2}}\left(\omega^{\prime}\right)\right\rangle_{c} \\
& \quad=2 \pi \delta\left(\omega+\omega^{\prime}\right) \int \frac{d v}{\pi} S_{x x-\mathrm{eq}}(\omega-v) S_{x x-\mathrm{eq}}(v) .
\end{aligned}
$$

Using this, we obtain

$$
\begin{aligned}
C(\omega)= & \frac{\varepsilon^{2} \kappa^{2}}{a^{2}} \int \frac{d v}{\pi} S_{x x-\mathrm{eq}}(\omega-v) S_{x x-\mathrm{eq}}(v) \\
& +S_{y y-\mathrm{eq}}(\omega-v) S_{y y-\mathrm{eq}}(v) \\
= & 2 \frac{\varepsilon^{2} \kappa^{2}}{a^{2}} \int \frac{d v}{\pi} S_{x x-\mathrm{eq}}(\omega-v) S_{x x-\mathrm{eq}}(v),
\end{aligned}
$$

the last line holding when the system is isotropic on the $(x, y)$ plane.
In order to compute $C(\omega)$, we note that the poles of $S_{x x-\mathrm{eq}}(v)$ are given by

$$
v_{p}=\frac{ \pm i \gamma \pm \sqrt{4 \kappa m-\gamma^{2}}}{2 m} .
$$

In the underdamped case where $4 \kappa m>\gamma^{2}$, the poles can be written as

$$
v=v_{+}, \quad v=\bar{v}_{+}, \quad v=v_{-}, \quad v=\bar{v}_{-},
$$

where

$$
\nu_{ \pm}=\frac{i \gamma \pm \sqrt{4 \kappa m-\gamma^{2}}}{2 m} .
$$

In the evaluation of the integral in Eq. (177), the poles that contribute from $S_{x x-\mathrm{eq}}(v)$ come from the upper complex plane and thus only $v=v_{ \pm}$contribute. The poles of $S_{x x-\mathrm{eq}}(\omega-v)$ can be written to be at $v-\omega=v_{p}$ and thus $v=v_{p}+\omega$. Therefore, only the poles $v=v_{ \pm}+\omega$ contribute from $S(\omega-$ $v)$. In terms of these poles, we can write

$$
S_{x x-\mathrm{eq}}(v) S_{x x-\mathrm{eq}}(\omega-v)=\frac{4 T^{2} \gamma^{2}}{m^{4}} \frac{1}{\left(v-v_{+}\right)\left(v-v_{-}\right)\left(v-v_{+}-\omega\right)\left(v-v_{-}-\omega\right)\left(v-\bar{v}_{+}\right)\left(v-\bar{v}_{-}\right)\left(v-\bar{v}_{+}-\omega\right)\left(v-\bar{v}_{-}-\omega\right)} .
$$

Evaluating the residues in the upper complex plane, we thus find

$$
\begin{aligned}
C(\omega)= & \frac{16 i \varepsilon^{2} \kappa^{2} T^{2} \gamma^{2}}{m^{4} a^{2}} \frac{1}{\left(v_{+}-v_{-}\right)(-\omega)\left(v_{+}-v_{-}-\omega\right)\left(v_{+}-\bar{v}_{+}\right)\left(v_{+}-\bar{v}_{-}\right)\left(v_{+}-\bar{v}_{+}-\omega\right)\left(v_{+}-\bar{v}_{-}-\omega\right)} \\
& +\frac{1}{\left(v_{-}-v_{+}\right)\left(v_{-}-v_{+}-\omega\right)(-\omega)\left(v_{-}-\bar{v}_{+}\right)\left(v_{-}-\bar{v}_{-}\right)\left(v_{-}-\bar{v}_{+}-\omega\right)\left(v_{-}-\bar{v}_{-}-\omega\right)} \\
& +\frac{1}{(\omega)\left(v_{+}+\omega-v_{-}\right)\left(v_{+}-v_{-}\right)\left(v_{+}+\omega-\bar{v}_{+}\right)\left(v_{+}+\omega-\bar{v}_{-}\right)\left(v_{+}-\bar{v}_{+}\right)\left(v_{+}-\bar{v}_{-}\right)} \\
& +\frac{1}{\left(v_{-}+\omega-v_{+}\right)(\omega)\left(v_{-}-v_{+}\right)\left(v_{-}+\omega-\bar{v}_{+}\right)\left(v_{-}+\omega-\bar{v}_{-}\right)\left(v_{-}-\bar{v}_{+}\right)\left(v_{-}-\bar{v}_{-}\right)} .
\end{aligned}
$$

We now proceed by writing

$$
v_{ \pm}=\frac{i \Gamma \pm \Omega}{2}
$$

where $\Gamma=\gamma / m$ and $\Omega=\sqrt{4 \kappa m-\gamma^{2}} / m$. Using this yields

$$
\begin{aligned}
C(\omega)= & \frac{16 i \varepsilon^{2} \kappa^{2} T^{2} \gamma^{2}}{m^{4} a^{2}} \frac{1}{\Omega(-\omega)(\Omega-\omega) i \Gamma(i \Gamma+\Omega)(i \Gamma-\omega)(i \Gamma+\Omega-\omega)} \\
& +\frac{1}{-\Omega(-\Omega-\omega)(-\omega)(i \Gamma-\Omega) i \Gamma(i \Gamma-\Omega-\omega)(i \Gamma-\omega)} \\
& +\frac{1}{\omega(\Omega+\omega) \Omega(i \Gamma+\omega)(i \Gamma+\Omega+\omega) i \Gamma(i \Gamma+\Omega)} \\
& +\frac{1}{(\omega-\Omega) \omega(-\Omega)(i \Gamma-\Omega+\omega)(i \Gamma+\omega)(i \Gamma-\Omega) i \Gamma} .
\end{aligned}
$$

This finally gives

$$
C(\omega)=\frac{64 \varepsilon^{2} \kappa^{2} T^{2} \gamma^{2}}{m^{4} a^{2} \Gamma\left(\Gamma^{2}+\Omega^{2}\right)} \frac{5 \Gamma^{2}+\omega^{2}+\Omega^{2}}{\left(\Gamma^{2}+\omega^{2}\right)\left[\Gamma^{2}+(\omega-\Omega)^{2}\right]\left[\Gamma^{2}+(\omega+\Omega)^{2}\right]} .
$$

Substituting this into Eq. (172) now gives

$$
S_{z z}(\omega)=2 T \gamma \frac{\left[1+\frac{32 \varepsilon^{2} \kappa^{2} T \gamma}{m^{4} a^{2} \Gamma\left(\Gamma^{2}+\Omega^{2}\right)} \frac{5 \Gamma^{2}+\omega^{2}+\Omega^{2}}{\left(\kappa \eta \omega^{2}\right)\left[\Gamma^{2}+(\omega-\Omega)^{2}\right]\left[\Gamma^{2}+(\omega+\Omega)^{2}\right]}\right]}{\left(\kappa \eta-m \omega^{2}\right)^{2}+\omega^{2} \gamma^{2}},
$$


which can be written as

$$
S_{z z}(\omega)=2 T \gamma \frac{\left[1+\frac{8 \varepsilon^{2} \kappa T}{m^{2} a^{2}} \frac{5 \Gamma^{2}+\omega^{2}+\Omega^{2}}{\left(\Gamma^{2}+\omega^{2}\right)\left[\Gamma^{2}+(\omega-\Omega)^{2}\right]\left[\Gamma^{2}+(\omega+\Omega)^{2}\right]}\right]}{\left(\kappa \eta-m \omega^{2}\right)^{2}+\omega^{2} \gamma^{2}}
$$

In terms of nearly all the physical parameters, we have

$$
S_{z z}(\omega)=\frac{2 T \gamma}{\left(\kappa \eta-m \omega^{2}\right)^{2}+\omega^{2} \gamma^{2}}\left[1+\frac{8 \varepsilon^{2} T \kappa}{a^{2}} \frac{4 \gamma^{2}+4 \kappa m+m^{2} \omega^{2}}{\left(\gamma^{2}+m^{2} \omega^{2}\right)\left(4 \kappa-2 \omega m \Omega+m \omega^{2}\right)\left(4 \kappa+2 \omega m \Omega+m \omega^{2}\right)}\right],
$$

where

$$
m \Omega=\sqrt{4 \kappa m-\gamma^{2}} .
$$

This can also be written as

$$
S_{z z}(\omega)=\frac{2 T \gamma}{\left(\kappa \eta-m \omega^{2}\right)^{2}+\omega^{2} \gamma^{2}}\left[1+\frac{8 \varepsilon^{2} T \kappa}{a^{2}} \frac{4 \gamma^{2}+4 \kappa m+m^{2} \omega^{2}}{\left(\gamma^{2}+m^{2} \omega^{2}\right)\left[\left(4 \kappa-m \omega^{2}\right)^{2}+4 \omega^{2} \gamma^{2}\right]}\right] .
$$

In the overdamped limit, where we set $m=0$, one finds

$$
S_{z z}(\omega)=\frac{2 T \gamma}{\kappa^{2} \eta^{2}+\omega^{2} \gamma^{2}}\left[1+\frac{8 \varepsilon^{2} T \kappa}{a^{2}} \frac{1}{4 \kappa^{2}+\omega^{2} \gamma^{2}}\right]
$$

which agrees with the calculation for an overdamped Brownian motion in Ref. [10].

In the case where the trap is also anisotropic on the $(x, y)$ plane, we write the stiffnesses in the directions $x, y$, and $z$ as $\kappa_{x}, \kappa_{y}$, and $\kappa_{z}$ and use the corresponding values of $w_{x}$ and $w_{y}$ for the fluctuating nonconservative force which we write from Eq. (29) as

$$
\mathbf{F}=\mathbf{F}_{x}+\mathbf{F}_{y}+\kappa \varepsilon a \mathbf{e}_{z}
$$

where

$$
\varepsilon=\frac{\kappa_{z} z_{0}\left(z_{0} k-1\right)}{\kappa a}
$$

with $a=\sqrt{a_{x} a_{y}}$ where $a_{x}=w_{x} / \sqrt{2}$ and $a_{y}=w_{y} / \sqrt{2}$. Here, $\kappa_{g}=\sqrt{\kappa_{x} \kappa_{y}}$ is the geometric mean of the stiffness in the directions $x$ and $y$. We can thus write

$$
\begin{aligned}
& \mathbf{F}_{x}=-\frac{\kappa_{g} \varepsilon_{x}}{a_{x}} x^{2} \mathbf{e}_{z}, \\
& \mathbf{F}_{y}=-\frac{\kappa_{g} \varepsilon_{y}}{a_{y}} y^{2} \mathbf{e}_{z},
\end{aligned}
$$

where $\varepsilon_{x}=\varepsilon a / a_{x}$ and $\varepsilon_{y}=\varepsilon a / a_{y}$. We can now simply use the results obtained above (as the contributions from $\mathbf{F}_{x}$ and $\mathbf{F}_{y}$ are additive) to obtain

$$
\begin{aligned}
S_{z z}(\omega)= & \frac{2 T \gamma}{\left(\kappa_{z}-m \omega^{2}\right)^{2}+\omega^{2} \gamma^{2}}\left[1+\frac{4 \varepsilon^{2} a^{2} \kappa_{g}^{2} T}{\kappa_{x} a_{x}^{4}} \frac{4 \gamma^{2}+4 \kappa_{x} m+m^{2} \omega^{2}}{\left(\gamma^{2}+m^{2} \omega^{2}\right)\left[\left(4 \kappa_{x}-m \omega^{2}\right)^{2}+4 \omega^{2} \gamma^{2}\right]}\right. \\
& \left.+\frac{4 \varepsilon^{2} a^{2} \kappa_{g}^{2} T}{\kappa_{y} a_{y}^{4}} \frac{4 \gamma^{2}+4 \kappa_{y} m+m^{2} \omega^{2}}{\left(\gamma^{2}+m^{2} \omega^{2}\right)\left[\left(4 \kappa_{y}-m \omega^{2}\right)^{2}+4 \omega^{2} \gamma^{2}\right]}\right] .
\end{aligned}
$$

If we denote the geometric mean of the two waists in the $x$ and $y$ directions by $w_{g}=\sqrt{w_{x} w_{y}}$, we find that, in terms of the waste variables, we have

$$
\begin{aligned}
S_{z z}(\omega)= & \frac{2 T \gamma}{\left(\kappa_{z}-m \omega^{2}\right)^{2}+\omega^{2} \gamma^{2}}\left[1+\frac{8 \varepsilon^{2} w_{g}^{2} \kappa_{g}^{2} T}{\kappa_{x} w_{x}^{4}} \frac{4 \gamma^{2}+4 \kappa_{x} m+m^{2} \omega^{2}}{\left(\gamma^{2}+m^{2} \omega^{2}\right)\left[\left(4 \kappa_{x}-m \omega^{2}\right)^{2}+4 \omega^{2} \gamma^{2}\right]}\right. \\
& \left.+\frac{8 \varepsilon^{2} w_{g}^{2} \kappa_{g}^{2} T}{\kappa_{y} w_{y}^{4}} \frac{4 \gamma^{2}+4 \kappa_{y} m+m^{2} \omega^{2}}{\left(\gamma^{2}+m^{2} \omega^{2}\right)\left[\left(4 \kappa_{y}-m \omega^{2}\right)^{2}+4 \omega^{2} \gamma^{2}\right]}\right] .
\end{aligned}
$$

If we consider the low-frequency limit $\omega \rightarrow 0$ and consider the simplest case where the system is isotropic on the ( $x, y$ ) plane, i.e., $w_{x}=w_{y}$, we find

$$
S_{z z}(0)=\frac{2 T \gamma}{\kappa^{2} \eta^{2}}\left[1+\frac{2 \varepsilon^{2} T}{a^{2}} \frac{\gamma^{2}+\kappa m}{\gamma^{2} \kappa}\right]
$$



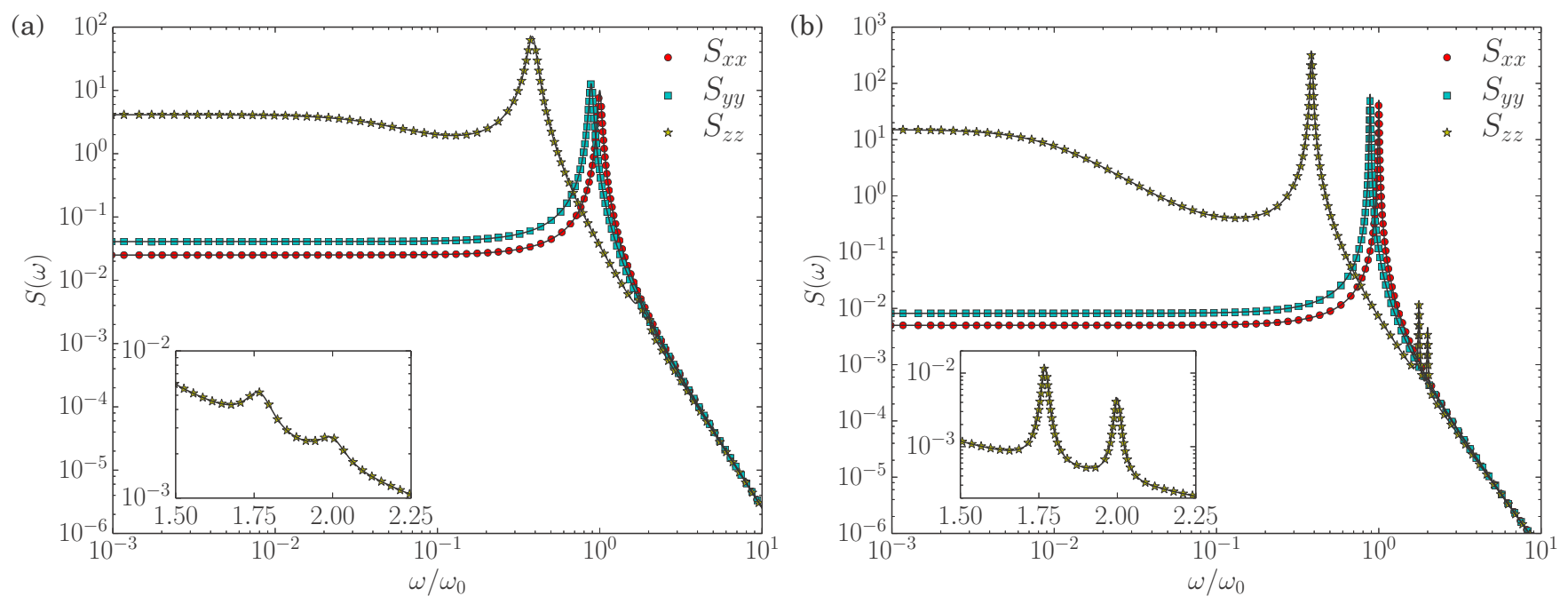

FIG. 4. Power spectrum for underdamped particles in the presence of scattering, obtained from numerical integration of Eqs. (155)-(157). The numerical simulations are plotted with symbols: $S_{x x}$ with disks, $S_{y y}$ with squares, and $S_{z z}$ with stars. The lines represent the exact equations for these functions, given by the general formula (197). The parameters taken here are $\eta_{y}=0.7828, \eta_{z}=0.1485, \sigma=0.5, \varepsilon=0.1$, and $Q=20$ for (a) and $Q=100$ for (b). In the insets, a zoom over the two peaks of $S_{z z}$ at $\omega=2 \omega_{0}$ and $\omega=2 \sqrt{\eta_{y}} \omega_{0} \simeq 1.77 \omega_{0}$ is represented.

which has the form

$$
S_{z z}(0)=\frac{2 T}{\kappa^{2} \eta^{2}}\left(\gamma A+\frac{B}{\gamma}\right)
$$

where $A$ and $B$ are damping-independent constants given by

$$
A=1+\frac{2 \varepsilon^{2} T}{\kappa a^{2}}, \quad B=\frac{2 \varepsilon^{2} T m}{a^{2}} .
$$

This predicts that $S_{z}(0)$ attains a minimal value at $\gamma_{c}=\sqrt{B / A}$.

\section{B. Signatures of time reversal symmetry breaking}

The crucial difference between equilibrium and nonequilibrium systems stems from the breaking of time reversal symmetry in the latter systems. It is this symmetry breaking that leads to the appearance of currents in nonequilibrium steady states [19]. Here, we show time reversal symmetry breaking can be inferred from temporal measurements of the particle positions.

Consider two observables $A$ and $B$ in a steady state; their correlation function is defined by

$$
C_{A B}(t)=\langle A(t) B(0)\rangle=\langle A(0) B(-t)\rangle=C_{B A}(-t),
$$

where the last step above uses the fact that any steady state by definition must be invariant by time translations [19].

The time reversal symmetry is broken when

$$
C_{B A}(t) \neq C_{B A}(-t),
$$

so that, in this case, the Onsager reciprocal relation [19],

$$
C_{A B}(t)=C_{B A}(t)
$$

does not hold.

For an equilibrium system as $C_{A B}(t)=C_{B A}(t)$, we find that $\tilde{C}_{A B}(\omega)$ is thus real. In the problem studied here, we need to find two operators $A$ and $B$ with nonzero correlation to demonstrate the violation of time reversal symmetry. The obvious choice $A=z$ and $B=x$ (or $B=y$ ) is not useful as the corresponding correlators are zero. However, choosing $B=x^{2}$ (or $B=y^{2}$ ) does yield a nonzero correlation function. However, to simplify the calculations which follow, we will choose $B=f_{n}(t)$, the fluctuating part of the component of the nonconservative force in the $z$ direction, which, see Eq. (164), is a function of $x^{2}$ and $y^{2}$ with zero mean, and from which the aforementioned correlators can be trivially extracted. We define the correlation function,

$$
C_{z f_{n}}(t)=\left\langle z(t) f_{n}(0)\right\rangle,
$$

which simplifies to

$$
C_{z f_{n}}(t)=\left\langle\zeta_{n}(t) f_{n}(0)\right\rangle,
$$

because $\zeta_{e f}$ is independent of $f_{n}$ and $f_{n}$ is chosen to have zero mean. This now gives

$$
\tilde{C}_{z f_{n}}(\omega)=\frac{C(\omega)}{-m \omega^{2}+i \gamma \omega+\kappa \eta},
$$

where $C(\omega)$ is given by Eq. (185). As $C(\omega)$ is real, we see that $\tilde{C}_{z f_{n}}(\omega)$ is not real and has an imaginary component,

$$
\tilde{C}_{z f_{n}}^{\prime \prime}(\omega)=\frac{-\gamma \omega C(\omega)}{\left(\kappa \eta-m \omega^{2}\right)^{2}+\gamma^{2} \omega^{2}} .
$$

Therefore, if one can experimentally measure the imaginary component of this correlation function, one has another method of probing the nonequilibrium steady state of trapped particles in optical traps.

\section{Numerical simulations}

Following Eqs. (155)-(157) where we have adimensionalized the space and time variables by writing them in units of $a$ and $\omega_{0}=\sqrt{\kappa_{X} / m}$, respectively, we compute the correlation function $\left\langle X(\omega) Y\left(\omega^{\prime}\right)\right\rangle=2 \pi \delta\left(\omega+\omega^{\prime}\right) S_{X Y}(\omega)$ from a fast Fourier transform of the time variable functions $X(t)$ 

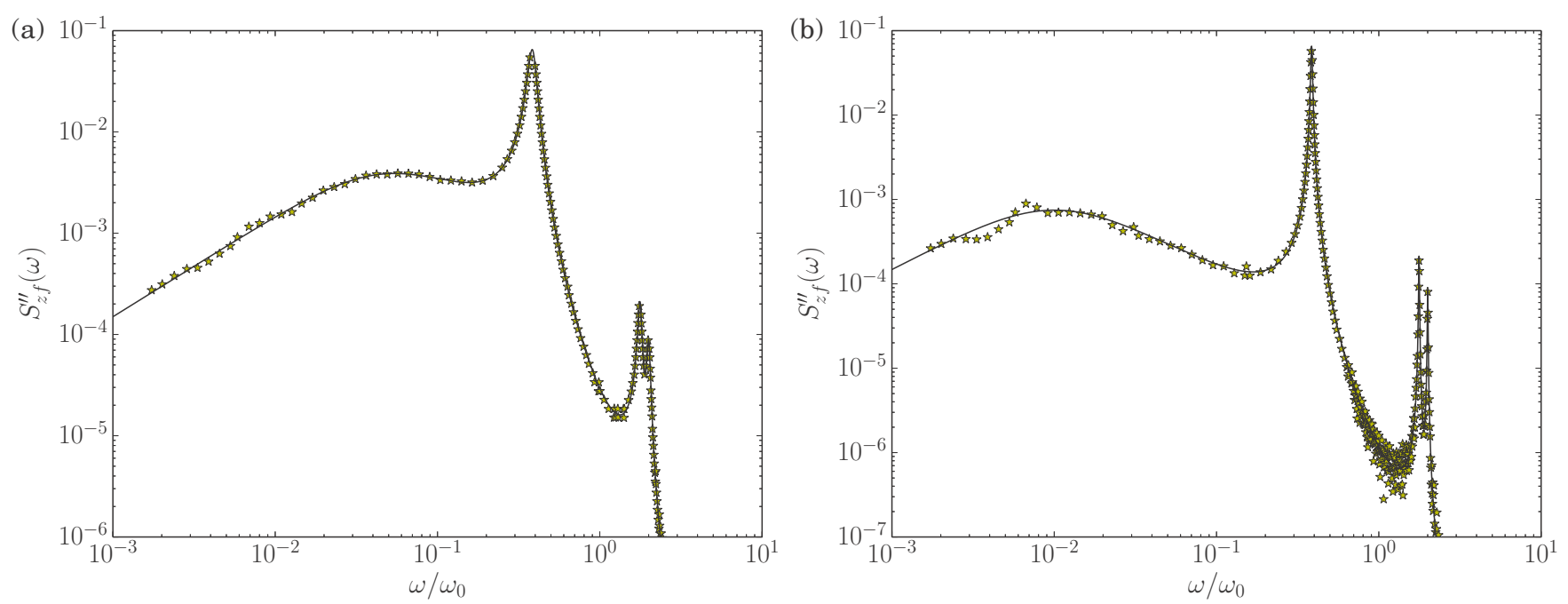

FIG. 5. The imaginary part of correlation function $S_{z f}(\omega)$ for the parameters (a) $\eta_{y}=0.7828, \eta_{z}=0.1485, \sigma=0.5, \varepsilon=0.1$, and $Q=$ 20 and (b) $Q=100$. The lines represent the exact Eq. (207), and the stars represent the numerical simulations with a stochastic average over 1000 realizations.

and $Y(t)$ over $2^{27} \sim 10^{8}$ points and $10^{3}$ stochastic averages where after discretization $S_{X Y}\left(\omega_{i}\right)=\left\langle X\left(\omega_{i}\right) Y^{*}\left(\omega_{i}\right)\right\rangle$ (where .$^{*}$ represents the complex conjugate). Shown in Fig. 4 is the numerical verification of the exact equation for $S_{z z}$ as predicted in Eq. (197). The time reversal symmetry breaking predicted by the nonzero value of $C_{z f}^{\prime \prime}$, given in Eq. (207), is verified by the results of the numerical simulations shown in Fig. 5.

\section{CONCLUSION}

Optical trapping can be carried out in both liquid and gas phases. In the gas phase, controlling the pressure can be used to modify the friction coefficient of the trapped particle as the gas viscosity changes. The fact that the system is subject to a nonconservative force means that the resulting steady state depends on the precise form of the dynamics, in this case, the damping coefficient $\gamma$. We have shown that this dependence on $\gamma$ can be demonstrated by measuring currents in the steady state, notably the currents associated with the marginal probability densities in both position and velocity space. Furthermore, the signature of optical scattering can be seen in the behavior of the spectral density function of the position and is particularly strong at low frequencies. We have also derived a correlation function between the particle position and the nonconservative force [equivalent to examining correlations between the position $Z(t)$ and the variable
$\left.X^{2}(t)\right]$ which has a component which does not respect time reversal symmetry, another measurement of nonequilibrium behavior.

Many of the nonequilibrium results derived here have a nonmonotonic dependence on the particle's friction coefficient and are thus susceptible to being optimized to maximize their potential experimental signals, pointing to directions for future experimental studies.

A promising future direction of this paper is to study the full nonlinear model for the optical trap to see what modifications are engendered by nonlinear effects. However, our numerical studies of the nonlinear model (see the accompanying paper [11]) suggest that the effects predicted by the harmonic model here persist and are thus robust. Finally, even within the harmonic model here, the nonlinear form of the conservative force means that we have only computed the first two moments of the temporal correlation functions and our results for the modified steady state distribution are perturbative. However, it would be interesting to compute the full distribution function even for this simple harmonic model.

\section{ACKNOWLEDGMENTS}

This work was partially funded by the Bordeaux IdEX Program-LAPHIA (Grant No. ANR-10-IDEX-03-02) and the ANR Project RaMaTRaF.
[1] A. Ashkin, Phys. Rev. Lett. 24, 156 (1970).

[2] P. Jop, J. Ruben G.-S., A. Petrosyan, and S. Ciliberto, J. Stat. Mech.: Theory Exp. (2009) P04012; D. G. Grier, Curr. Opin. Colloid Interface Sci. 2, 264 (1997); B. Lukić, S. Jeney, Ž. Sviben, A. J. Kulik, E.-L. Florin, and L. Forró, Phys. Rev. E 76, 011112 (2007).

[3] A. Ashkin and J. M. Dziedzic, Science 235, 1517 (1987); M. Wang, H. Yin, R. Landick, J. Gelles, and S. M. Block,
Biophys. J. 72, 1335 (1997); C. Bustamante, S. B. Smith, J. Liphardt, and D. Smith, Curr. Opin. Struct. Biol. 10, 279 (2000); J. M. Huguet, C. V. Bizarro, N. Forns, S. B. Smith, C. Bustamante, and F. Ritort, Proc. Natl. Acad. Sci. USA 107, 15431 (2010); J.-D. Wen, M. Manosas, P. T. X. Li, S. B. Smith, C. Bustamante, F. Ritort, and I. Tinoco, Jr., Biophys. J. 92, 2996 (2007); F. Ritort, S. Mihardja, S. B. Smith, and C. Bustamante, Phys. Rev. Lett. 96, 118301 (2006). 
[4] T. Li, S. Kheifets, and M. G. Raizen, Nat. Phys. 7, 527 (2011); J. Gieseler, B. Deutsch, R. Quidant, and L. Novotny, Phys. Rev. Lett. 109, 103603 (2012); M. Dienerowitz, M. Mazilu, and K. Dholakia, J. Nanophotonics 2, 021875 (2008); A. Lehmuskero, P. Johansson, H. Rubinsztein-Dunlop, L. Tong, and M. Käll, ACS Nano 9, 3453 (2015).

[5] D. Collin, F. Ritort, C. Jarzynski, S. B. Smith, I. Tinoco, and C. Bustamante, Nature (London) 437, 231 (2005); A. Bérut, A. Imparato, A. Petrosyan, and S. Ciliberto, Phys. Rev. Lett. 116, 068301 (2016); D. M. Carberry, J. C. Reid, G. M. Wang, E. M. Sevick, D. J. Searles, and D. J. Evans, ibid. 92, 140601 (2004); D. M. Carberry, M. A. B. Baker, G. M. Wang, E. M. Sevick, and D. J. Evans, J. Opt. A: Pure Appl. Opt. 9, S204 (2007).

[6] B. Sun, D. G. Grier, and A. Y. Grosberg, Phys. Rev. E 82, 021123 (2010).

[7] B. Sun, J. Lin, E. Darby, A. Y. Grosberg, and D. G. Grier, Phys. Rev. E 80, 010401(R) (2009).

[8] Y. Roichman, B. Sun, A. Stolarski, and D. G. Grier, Phys. Rev. Lett. 101, 128301 (2008).

[9] H. W. Moyses, R. O. Bauer, A. Y. Grosberg, and D. G. Grier, Phys. Rev. E 91, 062144 (2015).

[10] M. de Messieres, N. A. Denesyuk, and A. La Porta, Phys. Rev. E 84, 031108 (2011).

[11] Y. Amarouchene, M. Mangeat, B. V. Montes, L. Ondic, T. Guérin, D. S. Dean, and Y. Louyer, Phys. Rev. Lett. 122, 183901 (2019).
[12] W. Renz, Z. Phys. B: Condens. Matter 59, 91 (1985).

[13] S. A. Beresnev, V. G. Chernyak, and G. A. Fomyagin, J. Fluid Mech. 219, 405 (1990).

[14] T. Li and M. G. Raizen, Ann. Phys. (NY) 525, 281 (2013).

[15] J. Giesleler, L. Novotny, and R. Quidant, Nat. Phys. 9, 806 (2013).

[16] L. Novotny and B. Hecht, Principles of Nano-Optics, First ed. (Cambridge University Press, New York, UK, 2006).

[17] D. S. Dean, I. T. Drummond, and R. R. Horgan, J. Stat. Mech.: Theory Exp. (2007) P07013.

[18] P. Reimann, C. Van den Broeck, H. Linke, P. Hänggi, J. M. Rubi, and A. Pérez-Madrid, Phys. Rev. Lett. 87, 010602 (2001); B. Lindner, M. Kostur, and L. Schimansky-Geier, Fluct. Noise Lett. 01, R25 (2001); T. Guérin and D. S. Dean, Phys. Rev. Lett. 115, 020601 (2015); Phys. Rev. E 92, 062103 (2015); 95, 012109 (2017).

[19] R. Kubo, J. Phys. Soc. Jpn. 12, 570 (1957).

[20] B. Oksendal, Stochastic Differential Equations, 3rd ed. (Springer-Verlag, Berlin/Heidelberg, 1992).

[21] D. A. Sivak, J. D. Chodera, and G. E. Crooks, J. Phys. Chem. B 118, 6466 (2014).

[22] D. S. Dean, I. T. Drummond, R. R. Horgan, and S. N. Majumdar, Phys. Rev. E 71, 031103 (2005). 